\title{
Toni Pierenkemper
}

\section{Unternehmeraristokraten in Schlesien}

\section{Vorbemerkungen}

Die oberschlesischen Magnaten repräsentieren gegenüber den übrigen Gruppen des deutschen Adels offenbar einen ganz spezifischen Typus. Allerdings sollte man nicht dem Fehler anheimfallen, diesen Typus allein auf das deutsche Oberschlesien begrenzt zu sehen. Auch andernorts lassen sich „Magnaten“ antreffen, so z. B. in Teilen der Habsburgermonarchie ${ }^{1}$ und in anderen Regionen Ostmitteleuropas, so daß der vielbeschworene "Sonderfall“ Oberschlesien ${ }^{2}$ nicht bestätigt wird. Vielmehr zeigt sich hier eine heute besonders ausgeprägte Neigung der deutschen Historiographie, die Vergleichsobjekte nahezu ausschließlich aus Westeuropa zu wählen ${ }^{3}$ und den bis ins 20. Jahrhundert so bedeutsamen ost- und mittelosteuropäischen Raum weitgehend auszublenden ${ }^{4}$.

Der Begriff Magnat wird zur Benennung der herrschenden Oberschicht der ostmitteleuropäischen Aristokratie benutzt und leitet sich ursprünglich vom lat. „magnus“ ab. In der Adelsrepublik Polen bezeichnete man mit diesem Begriff die Angehörigen

1 Milan Myška, Die mährisch schlesische Eisenindustrie in der Industriellen Revolution (Prag 1970) und ein eindrucksvolles Beispiel bei Herbert Patzelt, Die Teschener Kammer. Geschichtliche Entwicklung der Teschener Kammergüter bis zum Ersten Weltkrieg, in: Oberschlesisches Jahrbuch (1991) 69-90. Zur Rolle des Adels als Unternehmer in Österreich vgl. auch Herbert Matis, Der österreichische Unternehmer. Erscheinungsbild und Repräsentanten, in: Karl-Heinz Manegold (Hrsg.), Wissenschaft, Wirtschaft und Technik. Studien zur Geschichte (München 1969) 286-298, hier $290 \mathrm{f}$. und Klaus Tenfelde, Unternehmer in Deutschland und Österreich während des 19. Jahrhunderts: Forschungsprobleme, in: Helmut Rumpler (Hrsg.), Innere Staatenbildung und gesellschaftliche Modernisierung in Österreich und Deutschland 1867/71 bis 1914 (Wien 1991) 125-138, insbes. 127.

2 So z. B. Rainer Fremdling, Technologischer Wandel und internationaler Handel im 18. und 19. Jahrhundert. Die Eisenindustrien in Großbritannien, Belgien, Frankreich und Deutschland (Berlin 1986) 141-150 und Hubert Kiesewetter, Industrielle Revolution in Deutschland 18151914 (Frankfurt/M. 1990) 187.

${ }^{3}$ So neuerdings Hartmut Kaelble, Vergleichende Sozialgeschichte des 19. und 20. Jahrhunderts: Forschungen europäischer Historiker, in: Jahrbuch für Wirtschaftsgeschichte (1993/1) 173-200.

${ }^{4}$ Unter Ausblendung der Verhältnisse in Oberschlesien und im Vergleich allein zur Ruhr vermag z. B. auch Richard van Dülmen einen saarländischen Sonderweg in die Industriewirtschaft zu konstatieren, der sich bei einer weitergreifenden Sicht der Dinge bald auflöst. Vgl. dazu $R i-$ chard van Dülmen, Sozialprofil der politischen Kultur. Der saarländische Weg der Modernisierung, in: ders. (Hrsg.), Industriekultur an der Saar. Leben und Arbeit in einer Industrieregion 1840-1914 (München 1989) 257-269. 
des Senatorenstandes, also die politische Oberschicht des Adels, die jedoch in rechtlicher Hinsicht von den übrigen Adelsangehörigen nicht geschieden war. Ähnlich wurde der Begriff auch in Ungarn verwandt, wo die Mitglieder der ersten, 1918 aufgelösten Kammer als Angehörige der sogenannten „Magnatentafel“ bezeichnet wurden". Vor diesem Hintergrund ist auch die Verwendung des Begriffs „Magnat" in Oberschlesien zu sehen: Hier wurde damit später die Gruppe der besonders einflußreichen großadeligen Industriellen beschrieben.

Diese finden sich nahezu ausschließlich in Oberschlesien, also in der am Oberlauf der Oder gelegenen wald-, erz- und kohlereichen Region, die später in preußischer Zeit ungefähr durch den Regierungsbezirk Oppeln umgrenzt wird. Niederschlesien, der größere und traditionsreichere Teil Schlesiens, weist demgegenüber eine gänzlich andere gewerbliche und demzufolge unternehmerische Tradition auf.

\section{Zum Typus des oberschlesischen Magnaten}

Wenn es auch Magnaten, d.h. adelige Eigentümer von industriellen Unternehmen, nicht nur in Oberschlesien gegeben hat, diese aber gerade für diese Region als besonders typisch gelten, so muß diese ungewöhnliche Wahrnehmung mit spezifischen Eigenarten dieser Gruppe in Schlesien zusammenhängen. Daher gilt es, die Entstehung, Entwicklung und Ausprägung dieser Personengruppe näher in den Blick zu nehmen. Möglicherweise war es ihr besonderes ökonomisches Engagement und damit verbunden ihr überdurchschnittlicher ökonomischer Erfolg, der gerade die oberschlesischen Magnaten derartig ins Blickfeld rückte.

Ihren Ursprung findet die eigentümliche Durchmischung von adeliger Gutswirtschaft und industriellem Unternehmertum bereits im 17. Jahrhundert, als die Grundherren in Oberschlesien dazu übergingen, die bis dahin „freien“ Hüttenmeister der Region in ihre Güterkomplexe einzugliedern. Dies geschah auf Initiative und durch Druck der Grundherren auf die zugewanderten Hüttenmeister, die in eigener Initiative die gewerblichen Ressourcen der Region erschlossen hatten. Insoweit profitierten die Grundherren von den unternehmerischen Initiativen der Hüttenmeister und erreichten durch die Eingliederung der gewerblichen Unternehmen eine Erweiterung ihrer ökonomischen Basis 6 .

In diesem Rahmen erfolgte nun eine extensive Ausdehnung der traditionellen, auf Luppenfeuern und Hammerwerken basierenden Eisenindustrie. Erze, Holz und Arbeitskräfte standen als herrschaftliche Ressourcen nahezu unbegrenzt zur Verfügung, und es entwickelte sich ein „System“ vorindustrieller Eisenproduktion, das über Jahr-

${ }^{5}$ Brockhaus Enzyklopädie, Bd. II (Wiesbaden 1970) 791.

6 Wactaw Dtugoborski, Die schlesischen Magnaten in der frühen Phase der Industrialisierung Oberschlesiens, in: Toni Pierenkemper (Hrsg.), Industriegeschichte Oberschlesiens im 19. Jahrhundert. Rahmenbedingungen - Gestaltende Kräfte - Infrastrukturelle Voraussetzungen - Regionale Diffusion (Wiesbaden 1992) 107-128, und umfassend ders., Ekonomika górnosiląskiego hutnictwa w XVIII wieku [Die Ökonomie des oberschlesischen Hüttenwesens im 18. Jahrhundert], in: Zeszyty Naukowe Wyższej Szkoly Ekonomicznej w Katowicach [Wissenschaftliche Hefte der Hochschule für Wirtschaft in Kattowitz] (1963). 
zehnte dem Grundherrn ein gesichertes Einkommen erbrachte. Das technologische Niveau der Produktion war jedoch niedrig und die Qualität der Produkte sehr gering.

Ein erster Modernisierungsschub setzte im frühen 18. Jahrhundert ein, als auch in Oberschlesien der Übergang der Eisenindustrie zum zweistufigen Eisengewinnungsverfahren mittels Holzkohlehochöfen und Frischfeuern erfolgte ${ }^{7}$. Hierbei spielten die Grundherren eine wichtige Rolle: Sie ergriffen die Initiative, warben Hüttenmeister vornehmlich aus dem weiter fortgeschrittenen Sachsen zur Anleitung der heimischen Arbeitskräfte an und setzten technische und organisatorische Neuerungen durch. So erfolgte der Übergang zur bergmännischen Gewinnung der Eisenerze, die zuvor als Raseneisenstein an der Erdoberfläche abgebaut worden waren. Arbeitskräfte wurden planmäßig rekrutiert und eine effizientere Absatzorganisation etabliert. Kurzum: Die Magnaten traten als aktive Unternehmer hervor und spielten eine wichtige Rolle bei der Modernisierung der Eisenindustrie in Oberschlesien im 18. Jahrhundert. 1756 finden sich daher auf den oberschlesischen Gütern 20 Holzkohlehochöfen und 43 Frischfeuer ${ }^{8}$.

Nach der Inbesitznahme Schlesiens durch Preußen veränderten sich die Rahmenbedingungen für die oberschlesische Industrie grundlegend ${ }^{9}$. Sehr bald wurde der preußische Staat selbst unternehmerisch aktiv und gründete in Malapane und Kreutzburg eigene Hüttenwerke, die sich jedoch weithin dem Muster der adeligen Werke anpaßten. Erst ab 1780 begann man auf den staatlichen Hütten mit der neuen „englischen“ Steinkohlentechnologie zu experimentieren und schließlich auch Kokshochöfen zu bauen und in Betrieb zu setzen. Diese Neuerung erwies sich jedoch entgegen der in der Geschichtsschreibung häufig aufzufindenden Meinung als zunächst wenig erfolgreich, und die Magnaten folgten dieser Entwicklung nur zögernd und in einem deutlichen zeitlichen Abstand ${ }^{10}$. Die Fortschritte im staatlichen Sektor zwangen die Magnaten jedoch langfristig zu Anpassungen an die nunmehr neueste Steinkohlentechnologie, um ihre Konkurrenzfähigkeit gegenüber den Anbietern außerhalb der Region und den staatlichen Hütten nicht zu verlieren. Sie büßten also die unternehmerische Initiative in diesem Sektor ein und wurden zur Anpassung gezwungen.

Nach 1815 expandierte der staatliche Sektor in Oberschlesien dann auf der Basis der neuen Technologien unter Einschluß des Steinkohlenbergbaus ganz außerordentlich. Zugleich wurden durch den Zinkboom der 1820er und 30er Jahre, der Oberschlesien kurzfristig an die Spitze der Weltzinkproduktion setzte, enorme Chancen für den Einstieg neuer, bürgerlicher Unternehmer in die Industrie Oberschlesiens geschaffen und von diesen auch wahrgenommen. Die Magnaten verhielten sich in dieser ersten starken, auf modernen Technologien beruhenden Expansionsphase der ober-

7 Dazu und zur weiteren Entwicklung der frühen Eisenindustrie vgl. Toni Pierenkemper, Das Wachstum der oberschlesischen Eisenindustrie bis zur Mitte des 19. Jahrhunderts - Entwicklungsmodell oder Spielwiese der Staatsbürokratie? in: ders. (Hrsg.), Industriegeschichte, 77-106.

B Dtugoborski, Die schlesischen Magnaten, 110.

9 Vgl. dazu Toni Pierenkemper, Grundzüge der Wirtschaftsgeschichte Oberschlesiens in der Neuzeit, in: ders. (Hrsg.), Industriegeschichte, 1-27.

10 Vgl. dazu Toni Pierenkemper, Das Wachstum, 97-99 und umfassend ders., Die privaten Hochofenwerke des oberschlesischen Industriereviers in der frühen Industrialisierung (1833-1856), in: Geschichte und Gesellschaft (voraussichtlich 1994). 
schlesischen Montanindustrie weitgehend passiv, da sie sich im alten Produktionssystem, basierend auf dem Einsatz ihrer grundherrlichen Ressourcen (gebundene Arbeit, Holz, Erz), gefangen sahen. Sie vermochten gegenüber den neuen technischen Herausforderungen relativ lange in diesem System zu verharren, weil sie die eingesetzten Ressourcen nicht mit ihren Marktpreisen zu kalkulieren brauchten und nur so mit effizienter arbeitenden, modernen Unternehmen konkurrieren konnten. Eine derartige Strategie mußte aber auf dynamischen Märkten sehr bald an ihre Grenzen stoßen und war langfristig zum Scheitern verurteilt.

Erst zur Mitte des 19. Jahrhunderts gelang den oberschlesischen Magnaten eine umfassende Modernisierung ihres Produktionssystems. Sie verfolgten nun eine aktive Industriepolitik und traten in offene Konkurrenz mit den bürgerlichen Unternehmern an Saar und Ruhr. Zugleich verloren sie als eigenständige Sozialgruppe an Bedeutung, trotz ihres Überganges zu moderner Technologie, des Ausbaus der Infrastruktur (Eisenbahnanschluß) und des Rückzuges des Staates aus der Montanindustrie. Innerhalb der Region verloren sie an Bedeutung gegenüber nichtadeligen Unternehmern und den sich ausbreitenden Aktiengesellschaften ${ }^{11}$, und nach außen verlor Oberschlesien an Bedeutung gegenüber dem sich nun weitaus dynamischer entwikkelnden Ruhrgebiet und der Saarregion ${ }^{12}$.

\section{Zum ökonomischen Erfolg der oberschlesischen Magnaten}

Die Ursachen für die bemerkenswerte Behauptung der oberschlesischen Grundherren beim Übergang von einer agrarwirtschaftlichen Produktionsweise zum industriellen Kapitalismus sind bis heute umstritten ${ }^{13}$. Zwei grundsätzliche Sachverhalte sind es vor allem, die zu einer Bewertung dieses unzweifelhaften ökonomischen Erfolges der oberschlesischen Magnaten herangezogen werden müssen.

Erstens stellt sich die Frage, ob ihr ökonomischer Erfolg nicht dadurch zu relativieren ist, daß er im wesentlichen in einer vorkapitalistischen, vormodernen Welt erzielt worden ist, in der die Verfügungsrechte über Wald, Bodenschätze und feudal gebundene Arbeitskräfte ausschlaggebend waren. Von einer marktwirtschaftlich organisierten, nach kapitalistischen Prinzipien betriebenen modernen Wirtschaftsweise kann in Oberschlesien im 17. und 18. Jahrhundert noch keineswegs gesprochen werden. Ökonomisch betrachtet, standen die Grundherren während des Auf- und Ausbaus der Magnatenwirtschaft im 17. und 18. Jahrhundert vor einer Situation, in der ihnen aufgrund ihrer an den Boden gebundenen Rechte ökonomische Ressourcen zufielen, die sie ohne Rücksicht auf die Gestehungskosten nutzen konnten: als „... eine Art Schatztruhe, eine unerwartete Vermehrung ihres durch Landbau und Viehzucht erworbenen

11 Zbigniew Kwaśny, Rozwój przemysłu na Górnym Śląsku w pierwszej polowie XIX wieku [Die Entwicklung der Industrie in Oberschlesien in der ersten Hälfte des 19. Jahrhunderts] (Breslau 1983) 38-47.

12 Toni Pierenkemper, Die industrielle Expansion an Saar, Ruhr und Oder. Die Zentren der deutschen Schwerindustrie im Vergleich, in: Jahrbuch für Wirtschaftsgeschichte (1992/1) 33-51.

${ }_{13}$ Vgl. dazu Dtugoborski, Die schlesischen Magnaten, 108. 
Vermögens ${ }^{\text {“14. }}$. In einer derartigen Konstellation können die mit solchen Ressourcen erstellten Produkte so lange unterhalb ihrer Gestehungskosten angeboten werden, als eine alternative Nutzung der Ressourcen nicht möglich ist. Dem Grundherrn stellt sich demnach nur die Frage, ob er z.B. seine feudal gebundenen Arbeitskräfte in seinen Bergwerken und Hütten arbeiten lassen oder ob er auf die Nutzung ihrer Arbeitskraft verzichten will, oder ob er z.B. das Holz aus seinem Waldbesitz zu Holzkohlen oder Grubenholz verarbeiten läßt oder der Verrottung preisgibt. Zahlreiche Rohstoffe und z.T. auch unqualifizierte menschliche Arbeitskraft bildeten innerhalb einer solchen Ökonomie quasi ein „freies Gut“, ihre Nutzung verursachte keinerlei Opportunitätskosten, weil sie alternativ gar nicht genutzt werden konnten.

Da Rohstoffe und Arbeitskräfte den Grundherren nahezu kostenlos zur Verfügung standen, waren sie in der Preisgestaltung ihrer Produkte weitgehend frei. Aus diesem Grunde war es für die oberschlesischen Magnaten auch bei geringer Arbeitsproduktivität sowie niedrigen Preisen und schlechter Qualität der Produkte profitabel, an den Markt zu treten, solange überhaupt nur ein Ertrag erwirtschaftet wurde: Die Kosten tendierten gegen Null, und ein Gewinn stellte sich daher quasi automatisch ein ${ }^{15}$.

Ob eine solche Produktionsweise insgesamt als ökonomisch erfolgreich angesehen werden kann, erscheint fraglich. Einzelwirtschaftlich betrachtet, ergibt sich daraus kurz- und vielleicht auch mittelfristig für die einzelnen Grundherren durchaus ein Gewinn, dem jedoch, langfristig und gesamtwirtschaftlich betrachtet, wegen der Unterbewertung der ökonomischen Ressourcen ein Verlust gegengerechnet werden muß. In einer solchen Ökonomie werden die Ressourcen eben nicht optimal genutzt und letztlich verschleudert. Sobald sich in einem solchen vorkapitalistischen Produktionssystem Konkurrenz um die Faktoren einstellt, d.h. bei der gewohnten Verwendung der Ressourcen Opportunitätskosten auftreten, und dies geschah in Oberschlesien, wie angedeutet, bereits im späten 18. Jahrhundert durch staatliche und bürgerlichindustrielle Initiativen, so stößt eine derart ressourcenintensive Produktionsweise an ihre Grenzen und kann sich bereits mittelfristig nicht mehr gegenüber der neuen, kapitalistischen Wirtschaftsweise behaupten.

Deshalb erscheint es zweitens hinsichtlich der Frage, ob die oberschlesischen Magnaten auch unter den neuen kapitalistischen Bedingungen im frühen 19. Jahrhundert ähnlich erfolgreich waren wie unter den alten, vorkapitalistischen Verhältnissen im 17. und 18. Jahrhundert, wichtig zu prüfen, inwieweit der Adel bei der Etablierung des kapitalistischen Wirtschaftssystems aktiv beteiligt war. Zweifel an einer führenden Rolle

${ }^{14}$ So David S. Landes, Der entfesselte Prometheus. Technologischer Wandel und industrielle Entwicklung in Westeuropa von 1750 bis zur Gegenwart (Köln 1973) 174.

is Auf den epochalen Charakter des Entstehens einer Marktwirtschaft weist bereits Karl Polanyi, The Great Transformation. Politische und ökonomische Ursprünge von Gesellschaften und Wirtschaftssystemen (Wien 1977, zuerst 1957) insbes. 58-62 hin. Die Logik ökonomischen Handelns ist wiederholt zum Gegenstand wissenschaftlicher Erörterungen gemacht worden. So z. B. bei Hans-Bernd Schäfer, Landwirtschaftliche Akkumulationslasten und industrielle Entwicklung. Analyse und Beschreibung entwicklungspolitischer Optionen in dualistischen Wirtschaften (Berlin 1983) und auch Dieter Grob, Strategien, Zeit und Ressourcen. Risikominimierung, Unterproduktivität und Mußepräferenz - die zentralen Kategorien von Subsistenzökonomien, in: Eberhard K. Seifert, Ökonomie und Zeit (Frankfurt am Main 1988) 131-188. 
sind angebracht, weil noch bis weit ins 19 . Jahrhundert hinein große Teile der grundherrlich zugehörigen Montanindustrie in die adelige Güterverwaltung integriert blieben und möglicherweise nicht als kapitalistische Unternehmen betrieben wurden ${ }^{16}$. Nur wenige führende Vertreter des Adels entwickelten sich zu Industriellen im engeren Sinne - und wenn, dann auch nur partiell und kurzzeitig, wie später noch zu zeigen sein wird ${ }^{17}$. Nicht sie, sondern vor allem der Staat entwickelte bei der Modernisierung der oberschlesischen Montanindustrie die größte Initiative ${ }^{18}$, der die Adeligen nur sehr zögernd folgten, z.T. einen vorsichtigen Kompromiß suchend zwischen der aufgezwungenen Modernisierung und dem Festhalten am vertrauten System. Und diese Strategie war zunächst auch ökonomisch rational, weil kurzfristig die Überlegenheit des neuen Systems sich noch gar nicht erwiesen hatte ${ }^{19}$. Als dann zur Mitte des 19. Jahrhunderts auch in Oberschlesien diese Überlegenheit offen sichtbar wurde, verschwanden dort die Relikte des alten Systems, die in die Güterverwaltungen integrierten, traditionell arbeitenden Montanbetriebe der Magnaten, schlagartig von der Bildfläche $^{20}$.

Dieser Anpassungsprozeß der oberschlesischen vormodernen Montanindustrie der Magnatenwirtschaft an die Bedingungen des kapitalistischen Produktionssystems vollzog sich etwa im Laufe eines halben Jahrhunderts (ca. 1790-1840) und nicht in allen Zweigen der Montanindustrie in gleicher Weise. Deshalb empfiehlt es sich, diesen Übergangsprozeß differenziert nach den Hauptbereichen dieser Branche zu untersuchen $^{21}$.

\subsection{Steinkohlenbergbau}

Hinsichtlich des Umfanges und der Entwicklung der Steinkohlenproduktion erweist sich wegen der besonderen politischen Geschichte Schlesiens das in Oberschlesien zunächst äußerst komplexe und uneinheitlich geregelte Bergrecht als wichtig ${ }^{22}$. Das

${ }^{16}$ Dlugoborski, Die schlesischen Magnaten, $108 \mathrm{f}$.

$17 \mathrm{Vgl}$. weiter unten unter Punkt 4 auf Seite $144 \mathrm{ff}$.

18 Aus der umfangreichen Literatur zu diesem Thema seien nur genannt William O. Henderson, The State and the Industrialization in Prussia (Liverpool 1958) 1-20, und Wolfhard Weber, Innovation im frühindustriellen Bergbau und Hüttenwesen. Friedrich Anton von Heynitz (Göttingen 1976).

19 Vgl. dazu Pierenkemper, Das Wachstum, und zur Frage der Effizienz einer Teilmodernisierung der Eisenindustrie in dieser Epoche generell Rainer Fremdling, Technologischer Wandel und internationaler Handel im 18. und 19. Jahrhundert. Die Eisenindustrien in Großbritannien, Belgien, Frankreich und Deutschland (Berlin 1896) 373-376.

20 Werner Bosack, Die Geschichte der Walzwerktechnik und die Entwicklung der Walzwerkindustrie im 19. Jahrhundert in Deutschland bis zur Wirtschaftskrise 1873 (Diss. Hannover 1970) 129, weist darauf hin, daß das Vorherrschen adeliger Montanbetriebe in Oberschlesien die Modernisierung der Industrie möglicherweise verzögert habe.

${ }^{21}$ Umfangreiches Material dazu bei Kwaśny, Rozwój przemysłu.

22 Umfassend dazu Albert Serlo, Beitrag zur Geschichte des schlesischen Bergbaus in den letzten hundert Jahren. Festschrift zur Feier des hundertjährigen Bestehens des Königlichen Oberbergamtes zu Breslau (Breslau 1860) 3-19, und knapp im Überblick Konrad Fuchs, Vom Dirigismus 
dann letztlich gültige Bergrecht von 1769 bevorrechtigte vor allem die Grundbesitzer, also den Adel. Dennoch befanden sich am Ende des 18. Jahrhunderts die Steinkohlengruben in Oberschlesien längst nicht mehr im Alleinbesitz der jeweiligen Grundherren, sondern mehrerer Personen, und auch Institutionen teilten sich z.T. den Besitz. Dies galt vor allem für die größeren und später erschlossenen Gruben, deren Finanzbedarf bereits so groß war, daß er die Möglichkeiten einzelner Grundherren überstieg.

Es bildeten sich daher im Steinkohlenbergbau Oberschlesiens bergrechtliche $\mathrm{Ge}$ werkschaften, deren Anteile, Kuxe genannt, sich relativ früh auch in Streubesitz bei bürgerlichen Gruppen fanden. In den Listen der Kuxeninhaber finden sich zahlreiche bürgerliche Berufsbezeichnungen wie Kaufmann, Bankier, Handwerker, Techniker und Arzt sowie auch eine Reihe nichtadliger Grundherren ${ }^{23}$. Der Kuxenbesitz war offenbar bereits in sehr hohem Maße mobil, denn es vollzog sich unter den Inhabern ein reger Kauf und Verkauf der Bergwerksanteile, d.h., es bildete sich bereits früh ein Markt für Kuxe heraus, der gewiß zur Herausbildung kapitalistischer Denk- und Verhaltensweisen wesentlich mit beitrug.

Der Absatz der Steinkohle in Oberschlesien blieb zunächst sehr begrenzt: Nach Mitte der 1830er Jahre wurde nur ca. ein Viertel der Förderung an die Zink- und Eisenhütten verkauft, drei Viertel mußten am Ort abgesetzt werden, weil ein Fernabsatz wegen fehlender Transportmöglichkeiten noch nicht möglich war. Die begrenzten Absatzmöglichkeiten bei der Hüttenindustrie finden ihre Erklärung in der Tatsache, daß in den großen Waldungen der Region mit dem Holz eine umfangreiche und preiswerte Energiebasis zur Verfügung stand, gegenüber der sich die Steinkohle zunächst nicht durchsetzen konnte. Noch zu Beginn des 19. Jahrhunderts wurden in Oberschlesien nur etwa 60 v.H. des Bestandzuwachses des Waldes als Nutzholz verwandt, der Rest verrottete ungenutzt ${ }^{24}$. Holz bildete auch noch zu jener Zeit offenbar keinen energetischen Engpaß für die ökonomische Expansion der Region, wenngleich bereits ein Rückgang der Waldfläche und ein allmählicher Anstieg der Holzpreise eine Annäherung der Holznachfrage an das Angebot in näherer Zukunft erwarten lassen konnten. Eine weitere Expansion des Energieverbrauchs auf der Grundlage des heimischen Holzes schien zunächst noch gesichert, und erst in den 1840er Jahren näherte sich dann auch der Energieverbrauch der Region der Leistungsgrenze des dortigen Waldes. Dennoch erlebte die Steinkohlenförderung in Oberschlesien bereits im frühen 19. Jahrhundert einen ersten Aufschwung: 1815 wurde dauerhaft die $100000-t-G r e n z e$ der Jahresförderung überschritten ${ }^{25}$. Diese frühe Expansion der Steinkohlenförderung folgte ganz wesentlich dem Aufschwung der oberschlesischen

\section{Fortsetzung Fußnote von Seite 134}

zum Liberalismus. Die Entwicklung Oberschlesiens als preußisches Berg- und Hüttenrevier (Wiesbaden 1970) $206 \mathrm{f}$.

${ }_{23}$ Kwaśny, Rozwój przemystu, 38.

${ }^{24}$ Schätzung nach $A$. Nyrek, Gospodarka leśna na Górnym Śląsku od pot. XVII do poł. XIX w. [Die Forstwirtschaft in Oberschlesien von der Mitte des 17. bis zur Mitte des 19. Jahrhunderts] (Breslau 1975).

${ }^{25}$ Vgl. zu den Produktionszahlen die Tabelle im Anhang. 
Zinkindustrie, die einen ersten Höhepunkt im Zinkboom von 1815-1823 erlebte und dann nochmals in einem zweiten Boom in den 1840er Jahren ihre Produktion und ihren Absatz deutlich steigern konnte ${ }^{26}$. Während dieser Phase verlagerte sich die Steinkohlenproduktion dabei von den kleineren Gruben zu den neuen, größeren Schachtanlagen im schlesischen Zentralrevier um Beuthen ${ }^{27}$.

Die Expansion der Steinkohlenproduktion ging einher mit dem Aufbau einer ausdifferenzierten Vertriebsstruktur und der Ausdehnung des Absatzgebietes. Zunächst blieb jedoch der Absatz wegen fehlender Transportmöglichkeiten noch lokal begrenzt, doch wurden Händler, Märkte und Messen zunehmend genutzt. Erst zur Mitte des Jahrhunderts, nach Fertigstellung des Eisenbahnanschlusses ${ }^{28}$, konnten größere Kohlenmengen über den Schienenweg abgesetzt werden: 1855 waren es 27,2 v. H.

Der Betrieb von Steinkohlenbergwerken erwies sich in Oberschlesien wie andernorts als relativ kapitalintensiv. Den Hauptkostenbestandteil für die Produktion bildeten mit 40-50 v.H. die sogenannten Abbaukosten, in denen neben den Löhnen der Bergleute vor allem die Kosten des Grubenausbaus enthalten waren. Für die Beamtenund Aufseherarbeiten waren lediglich $10 \mathrm{v} . \mathrm{H}$. anzusetzen und für Gebühren und Steuern etwa 20 v. H. Der Rest der Produktionskosten wurde durch Nebenkosten und Sonderausgaben verursacht ${ }^{29}$. Die Differenz zwischen den Gewinnungskosten und dem Abgabepreis (Taxe) war beachtlich; offenbar handelte es sich beim Steinkohlenbergbau um einen höchst profitablen Erwerbszweig.

\subsection{Zinkindustrie}

Die große Nachfrage nach Zink und die in Oberschlesien reichlich verfügbaren Rohund Brennstoffe sowie die im Vergleich zu Eisenhütten relativ geringen Anforderungen an technisches Know-how und maschinelle Ausstattung, was bedeutende Gewinnspannen erlaubte, führten ab der zweiten Hälfte der zwanziger Jahre zu einem Gründungsboom in diesem Gewerbezweig mit einem enormen jährlichen Zuwachs an Hütten ${ }^{30}$. Im 19. Jahrhundert wurde Rohzink nahezu ausschließlich aus dem zunächst im Tagebau gewonnenen Galmei geröstet ${ }^{31}$. Noch in den 90er Jahren, als sich die oberschlesischen Galmeilager zusehends erschöpften, hatte die nun in den oberschlesischen Zinkhütten verstärkt genutzte bergmännisch gewonnene Zinkblende

${ }^{26}$ Zum Zinkboom vgl. Fuchs, Vom Dirigismus, 99-116, und Norman Pounds, The Upper Silesian Industrial Region (s'Gravenhage 1958) 81 ff., und Kwaśny, Rozwój przemysłu, 111-114.

27 Zur kleinräumlichen Gliederung des oberschlesischen Industriebezirks vgl. Pierenkemper, Die privaten Hochofenwerke.

${ }_{28}$ Dazu ausführlich Fucbs, Vom Dirigismus, 171-238.

29 Kwaśny, Rozwój przemyslu, 190. Die Angaben beziehen sich auf eine Reihe verschiedener Gruben für das Jahr 1817.

${ }^{30}$ Kwaśny, Rozwój przemyslu, 111 f.; Jan S. Dworak, Karol Godula - pionier przemystu cynkowego na Górnym Śląsku [Karl Godulla - ein Pionier der Zinkindustrie in Oberschlesien] (Oppeln 1979) 51, 54.

31 Ausführlich dazu Pounds, The Upper Silesian, 81-96. 
erst einen Anteil von 31,5 v.H., wobei allerdings das Galmei zu dieser Zeit schon vorwiegend von den Halden genommen wurde ${ }^{32}$.

Bei der größten und ertragreichsten Scharley-Grube waren es die Familie Donnersmarck/Beuthen-Siemianowitz und Alexander Schreiber, die den größten Anteil auf sich vereinigten ${ }^{33}$. Bei den Zinkhütten waren die Eigentumsverhältnisse insgesamt noch weiter gestreut als bei den Galmeigruben. Hier dominierten zunächst nicht-adelige Unternehmer die Entwicklung; erst später wandten sich dann auch die adeligen Familien diesem Sektor zu. Die folgende Tabelle veranschaulicht die Anteile der wichtigsten Zinkproduzenten an der Produktion in der ersten Hälfte des 19. Jahrhunderts.

Prozentualer Anteil der Zinkhütteninhaber an der oberschlesischen Zinkproduktion (1822$1856)^{34}$

\begin{tabular}{llllll}
\hline Produzent & 1822 & 1833 & 1843 & 1853 & 1856 \\
\hline Pastor Naglo & 16,9 & - & - & - & - \\
Baildon & 16,0 & - & - & - & - \\
Ballestrem & 14,9 & - & - & - & - \\
Inspektor Harnisch & 14,1 & - & - & - & - \\
Giesche Erben & 13,6 & 23,2 & 13,4 & 11,7 & 12,1 \\
Sth. Donnersmarck & - & 22,9 & - & - & - \\
Kaufmann Lüschwitz & - & 12,2 & 21,2 & - & - \\
Winckler (von Tiele-Winckler) & - & 11,7 & 15,2 & 19,1 & 17,6 \\
Griebel & - & 10,8 & - & - & - \\
Schneider & - & - & 9,5 & - & - \\
Heinitze & - & - & 9,5 & - & - \\
Graf Hugo H. von Donnersmarck & - & - & - & 11,0 & 11,6 \\
Graf H. von Donnersmarck & - & - & - & 11,9 & - \\
Gryzik & - & - & - & 10,3 & 15,3 \\
Schlesische Actiengesellschaft & - & - & - & - & 9,6 \\
\hline Insgesamt & 75,5 & 80,8 & 68,8 & 64,0 & 65,2 \\
\hline
\end{tabular}

Aus den Zahlen wird deutlich, daß bürgerliche Unternehmer diesen expansivsten und profitabelsten Sektor der oberschlesischen Montanindustrie recht lange dominierten. Auch die Technologie in diesem Produktionszweig entsprach dem neuesten Stand: Man gewann dort das Rohzink durch das Rösten der Erze mittels Steinkohlen in neu errichteten Röstöfen ${ }^{35}$. Einen beispiellosen Erfolg in dieser Branche erzielte

${ }^{32}$ Vgl. Kazimierz Popiotek, Górnośląski przemysł górniczo-hutniczy w drugiej połowie XIX wieku [Die Berg- und Hüttenindustrie in Oberschlesien in der zweiten Hälfte des 19. Jahrhunderts] (Kattowitz 1965) $154 \mathrm{ff}$.

33 Fuchs, Vom Dirigismus, 108; Kwaśny, Rozwój przemysłu, 39.

${ }_{34}$ Kwaśny, Rozwój przemysiu, 45 f.

${ }^{35}$ Giesches Erben, während eines Jahrhunderts bis 1802 Inhaber des Galmeiprivilegs, verlagerten wegen des großen Kohlenbedarfs der Röstöfen Anfang der 20er Jahre den Standort ihrer Zinkhütten in die Umgebung von Steinkohlengruben: 1818 Georg-Hütte bei der Grube Fanny 
hier der „Zinkkönig“ Godulla, dessen Besitztümer über seine Erbin Johanna Gryzik letztendlich dem Grafen Schaffgotsch zufielen ${ }^{36}$. Zunehmend wurde auch der Handel mit Zinkerzen wichtiger, und mehr und mehr gewannen Kaufleute in der Zinkbranche an Bedeutung, so daß sich das an sich schon recht weite unternehmerische Rekrutierungsfeld der Zinkindustrie noch stärker ausdehnte.

Das Eigentum an den Galmeigruben und an den Zinkhütten wechselte in Oberschlesien relativ häufig den Besitzer. Die Branche erwies sich zwar als hochprofitabel, aber instabil und gewährte daher spekulativen Geschäften weiten Raum. Zur Minderung des individuellen Risikos gingen daher die Unternehmer dieses Gewerbezweiges schon relativ früh zur Bildung von Mehrpersonen-Unternehmen über, und sie trugen damit ebenfalls zur Verbreitung kapitalistischer Denk- und Verhaltensweisen in dieser eher rückständigen Region bei. Die deutliche Expansion der Zinkproduktion nach 1815 und besonders nach $1820^{37}$ förderte ganz entscheidend die Steigerung der Nachfrage nach Steinkohlen und damit die Entwicklung des Steinkohlenbergbaus in Oberschlesien. Die Zinkindustrie bildete in den 1820er Jahren den Hauptnachfrager für den oberschlesischen Steinkohlenbergbau. Im Unterschied zum Steinkohlenbergbau erforderte der Galmeibergbau jedoch nur geringe Vorleistungen und war daher äuBerst profitabel, profitabler noch als der Steinkohlenbergbau und der Betrieb von Zinkhütten. Bei den Zinkhütten war der Hauptkostenbestandteil der Rohstoff (Galmei), der etwa 40-50 v. H. aller Aufwendungen erforderlich machte, gefolgt von 20-30 v. H. für den Brennstoff (Steinkohlen) und $10 \mathrm{v}$. H. für Transporte (Fuhrlohn). Alle anderen Hüttenkosten, einschließlich der Löhne für die Hüttenarbeiter, waren in den restlichen Kosten enthalten. Offenbar arbeitete man innerhalb dieser rohstoffintensiven Industrie mit sehr geringen Fixkosten ${ }^{38}$, was eine relativ leichte Anpassung an wechselnde Konjunkturen und hohe Gewinne ermöglichte.

\subsection{Eisenhüttenwesen}

Der Eisenerzbergbau in Oberschlesien befand sich zu Beginn des 19. Jahrhunderts noch nahezu vollständig in adeligem Besitz. Eine Ausnahme bildeten lediglich der Pastor Naglo aus Tarnowitz und die Stadt Beuthen, die ebenfalls Förderrechte an Eisensteinfeldern besaßen. 1822 wurde insgesamt in 27 oberschlesischen Ortschaften Eisen-

Fortsetzung Fußnote von Seite 137

bei Michałkowice, 1825 Erwerb der Lieres-Hütte im Beuthener Wald und Bau der David-Hütte unter Eröffnung der König-Saul-Grube bei Chropaczów (vgl. Jerzy Jaros, Historia firmy „Giesche" [Die Geschichte der Firma "Giesche"], in: Wiadomości hutnicze [Hütten-Nachrichten] 43 (1987) 7 f., 204; Geschichte der Bergwerksgesellschaft Georg von Giesches Erben, Bd. 1: Konrad Wutke, Allgemeine Geschichte, 207: Józef Piernikarczyk, Historia górnictwa i hutnictwa na Górnym Ślạsku [Geschichte des Bergbaus und des Hüttenwesens in Oberschlesien] t.2 (Kattowitz 1936) 54-58.

${ }^{36} \mathrm{Zu}$ Karl Godulla vgl. weiter unten unter Punkt 4 auf Seite 150-151.

${ }^{37}$ Vgl. die Produktionstabelle im Anhang auf Seite 157.

${ }^{38}$ Der Umfang der Fixkosten in den Unternehmen der frühen Industrialisierung wird zumeist weit überschätzt. Vgl. dazu Sidney Pollard, Fixed Capital in the Industrial Revolution, in: JEH (1964/3) 299. 
erz gefördert ${ }^{39}$. Ab den 1830er Jahren erfolgte dann eine sukzessive Erschließung des Eisenerzbergbaus durch bürgerliche Gruppen, hinzu traten Vertreter des Kleinbürgertums und schließlich sogar einige Bauern, die sich ebenfalls diesem Erwerbszweig zuwandten. Von den 1850er Jahren an fanden auch hier dann Aktiengesellschaften stärkere Verbreitung.

Das starke Interesse des Adels am Eisenerzbergbau läßt sich auf die produktionstechnischen Vorteile zurückführen, die bei einer Verhüttung eigener Erze im Hochofen auftraten. Die reichen Erzlager, die sich vor allem in der Gegend um Tarnowitz fanden, wurden allerdings nicht dort, sondern am Fundort der Kohle, die inzwischen auch für die Eisenindustrie an Bedeutung gewonnen hatte, also im Gebiet um Beuthen verhüttet.

Die Familie Winckler, die unter den Privateigentümern an Eisenerzgruben zur Jahrhundertmitte mit den beiden Donnersmarcklinien und dem Grafen Renard die Spitzenposition innehatte ${ }^{40}$, repräsentiert hier einen besonders erfolgreichen Fall bürgerlicher unternehmerischer Initiative im Bereich der Gewinnung und Verhüttung von Eisenerzen ${ }^{41}$. Auch zahlreiche andere Kaufleute suchten Zugang zum Erzbergbau, ohne jedoch zugleich Hütten zu erwerben oder neue zu errichten. Bürger der Städte Myslowitz, Tarnowitz, Beuthen und Gleiwitz taten sich dabei besonders hervor. Zu nennen sind hier die Bankiers Moritz und Simon Friedländer sowie Simon Levi ${ }^{42}$, die großen Einfluß auf den Handel mit Eisenerzen gewannen. Dieser Handel war offenbar höchst einträglich und griff in Oberschlesien immer weiter um sich, so daß sich schließlich selbst Beamte der preußischen Berg- und Hüttenverwaltung daran beteiligten.

\subsubsection{Roheisengewinnung}

Die Umstellung der Eisenherstellung auf das moderne „englische“ Kokshochofenverfahren erfolgte in Oberschlesien erst ab den 1830er Jahren, obwohl bereits am Ende des 18. Jahrhunderts auf staatliche Initiative hin erstmals derartige Versuche unternommen worden waren. $\mathrm{Zu}$ diesem Zeitpunkt stellten die Grundherren immer noch die bedeutendste Gruppe in der Unternehmerschaft dieser Branche, wenn auch ihre

39 Kwaśny, Rozwój przemyslu, 39; ders., Hutnictwo żelaza na Górnym Śląsku w pierwszej potowie XIX wieku [Das Eisenhüttenwesen in Oberschlesien in der ersten Hälfte des 19. Jahrhunderts] (Breslau 1968) 24-29.

40 Vgl. Popiotek, Górnośląski przemysł, 24.

41 Vgl. Güntber Grundmann, Friedrich Wilhelm Grundmann (Augsburg 1956) 130-136; zur Person Wincklers: Konrad Fuchs, Franz von Winckler, in: Schlesier des 15.-20. Jahrhunderts, Bd. V (Würzburg 1968) 107-112; ders., Franz von Winckler, in: Beiträge zur Wirtschafts- und Sozialgeschichte Schlesiens (Dortmund 1985) 117-122.

${ }^{42}$ Zum Einfluß des jüdischen Unternehmertums in Oberschlesien vgl. auch Toni Pierenkemper, Jüdische Unternehmer in der deutschen Schwerindustrie - Vexierbild oder Chimäre?, in: Werner E. Mosse u. Hans Pobl, Jüdische Unternehmer in Deutschland im 19. und 20. Jahrhundert (Stuttgart 1992) 100-118, und ders., Jüdische Industrielle in Oberschlesien im 19. Jahrhundert, in: Jahrbuch der Schlesischen Friedrich Wilhelms Universität zu Breslau, Bd. 32 (1991) 197-220, ebenso Kúániny, Hutnictwo żelaza, 24. 
Bedeutung allmählich zu schwinden begann. Nicht-adelige Grundbesitzer und bürgerliche Gruppen holten auf, ohne jedoch die Dominanz des Adels zunächst brechen zu können.

Anteil der Hochofeneigentümer in Oberschlesien (1822-1856) nach Zugehörigkeit (in v. H.) ${ }^{43}$

\begin{tabular}{lrrrrrr}
\hline Besitzer & 1822 & 1833 & 1839 & 1846 & 1851 & 1856 \\
\hline Staat & 15,6 & 13,7 & 11,1 & 10,7 & 8,6 & 8,9 \\
Adel & 80,0 & 80,4 & 77,0 & 73,1 & 68,5 & 63,9 \\
Grundbesitzer ohne Adelstitel & 4,4 & 3,9 & 9,5 & 7,7 & 14,8 & 3,8 \\
Bürger, Kaufleute, Bankiers & - & - & - & 4,6 & 2,5 & 3,8 \\
Aktiengesellschaft & - & - & - & - & - & 8,9 \\
Unbekannt & - & 2,0 & 2,4 & 3,8 & 5,6 & 10,7 \\
\hline Insgesamt & 100,0 & 100,0 & 100,0 & 100,0 & 100,0 & 100,0 \\
\hline
\end{tabular}

Die Eigentumsverhältnisse in der Hochofenindustrie in Oberschlesien veränderten sich, wie die Zahlen der obigen Tabelle verraten, nur sehr allmählich. Eine Reihe der nicht-adeligen Grundbesitzer wurden später zudem nobilitiert (z.B. Winckler), so daß auf diese Weise dem schwindenden Einfluß des Adels auf die Industrie entgegengewirkt wurde. Die dominante Stellung des Adels im Eisenhüttenwesen Oberschlesiens im 19. Jahrhundert beruhte auf der Tatsache, daß unter den Bedingungen der alten, vorindustriellen Ökonomie der Betrieb von Hochöfen nur im Zusammenhang mit der Verfügung über grundherrliche Ressourcen möglich bzw. ertragreich war ${ }^{44}$. Erst von den 1830er Jahren an, nach Herstellung kapitalistischer Bedingungen in diesem Wirtschaftszweig, wurden Investitionen darin auch für bürgerliche Gruppen interessant.

Praktisch vollzog sich dieses bürgerliche Engagement im Eisenhüttenwesen in enger Kooperation zwischen bürgerlichen Financiers und adeligen Grundbesitzern. Der Berliner Maschinenfabrikant Franz Anton J. Egells z.B. errichtete mit dem Grafen Detlef von Einsiedel, Metallwerkbesitzer zu Lauchhammer, die Eintrachtshütte im Beuthener Wald ${ }^{45}$. Die Familie Donnersmarck (Beuthen-Siemianowitz) wurde bei der Gründung der Laura-Hütte durch die Gebrüder Oppenfeld unterstützt ${ }^{46}$. Nach dem von Wedding ${ }^{47}$ ausgearbeiteten Investitionsplan finanzierten beide Seiten je zur Hälfte diese hochmoderne, unter Beteiligung von Nottebohm errichtete Anlage, wel-

43 Kwaśny; Rozwój przemysłu, 43.

44 Vgl. weiter oben auf Seite 132-133.

45 Zu Egells: Alfons Perlick, Franz Anton J. Egells, in: Oberschlesische Berg- und Hüttenleute (Kitzingen 1953) 201; Kurt Groba, Franz Anton Egells, in: Der Südosten 19 (1940) 628 f.; Walter Döring, Gerbard Schmidt, Detlef Graf Einsiedel, in: NDB, Bd. 4 (1959) $400 \mathrm{f}$.

46 Margarete Czaja, Der industrielle Aufstieg der Beuthen-Siemianowitzer und Tarnowitz-Neudecker Linie der Henckel von Donnersmarck bis zum Weltkrieg (München 1936) 38; Kwaśny, Hutnictwo żelaza, 38; ders., Rozwój przemysłu, 44.

$47 \mathrm{Zu}$ Wedding vgl. knapp Fuchs, Vom Dirigismus, 138, FN 64. 
che die riesige Summe von 350000 Talern verschlang, wobei der den Boden zur Verfügung stellende Magnat innerhalb von 22 Jahren den Anteil der Bankiers ablöste ${ }^{48}$. Die bürgerlichen Investoren kauften zumeist bestehende Hochofenwerke auf oder beteiligten sich daran. Die 1840 von den Bankiers Moritz Friedländer (Gleiwitz), Simon Levi (Beuthen) und David Löwenfeld (Breslau) ${ }^{49}$ im Beuthener Wald gegründete Friedens-Eisenhütte war die erste Gründung eines Hochofenwerkes allein durch bürgerliche Unternehmer, der weitere folgten, z. B. die des Geheimen Kommerzienrats Löbbecke aus Breslau und Viktor Laband. Die ohne eigene Rohstoffbasis am Ende der guten Eisenkonjunktur entstandene Friedens-Eisenhütte kam bald in Schwierigkeiten und wurde schließlich 1851 von Graf Renard übernommen, der damit neben Hugo Henckel zum bedeutendsten Eisenproduzenten Oberschlesiens aufstieg ${ }^{50}$.

Im Verbund mit bürgerlichen Kapitalgebern beherrschten also in den 1840er Jahren die adeligen Grundherren die oberschlesische Roheisenproduktion. Innerhalb der Branche fanden sich zwar die verschiedenartigsten Kombinationen, eine grundsätzliche Umverteilung des Eigentums an den Eisenhütten erfolgte jedoch nicht. Eine Analyse der Verkäufe von Hochofenwerken zwischen 1822 und 1856 zeigt, daß die überwiegende Mehrheit der Eigentumsübertragungen innerhalb des Adels selbst erfolgte. Bürgerliche Gruppen waren daran so gut wie nicht beteiligt.

Eigentumswechsel von Hochofenwerken (1822-1856) in Oberschlesien ${ }^{51}$

\begin{tabular}{lcc}
\hline Soziale Stellung des Eigentümers & übergebende & übernehmende \\
\hline Adel & 27 & 17 \\
Grundbesitzer ohne Adelstitel & 2 & 3 \\
Andere & 2 & 7 \\
Unbekannt & 1 & 5 \\
\hline Insgesamt & 32 & 32 \\
\hline
\end{tabular}

Zwar verkaufte der Adel häufiger, als daß er kaufte, und er mußte so einen gewissen Bedeutungsverlust hinnehmen. Die Dominanz der Grundherren wurde damit jedoch längst noch nicht in Frage gestellt. Zwischen 1822 und 1850 standen in wechselnder Reihenfolge Renard, Hohenlohe auf Slawentzitz und Donnersmarck auf Beuthen-Siemianowitz mit deutlichem Abstand an der Spitze der Roheisenproduktion ${ }^{52}$.

\footnotetext{
${ }^{48}$ Jerzy Jaros, Tajemnice górnośląskich koncernów [Die Geheimnisse der oberschlesischen Konzerne] (Kattowitz 1988) 54-57.

49 Konrad Fuchs, Zur Rolle des Judentums in der Wirtschaft Oberschlesiens, in: Zeitschrift für Ostforschung 28 (1979) 276, und Pierenkemper, Jüdische Industrielle.

so Konrad Fuchs, Andreas Maria Graf Renard (1795-1875) und seine Bedeutung für die oberschlesische Industrie, in: ders., Beiträge, 107-116, S. 113.

${ }^{51}$ Kwaśny, Rozwój przemystu, 44.

s2 Vgl. Kwaśny, Hutnictwo, 39.
} 
Die Roheisenproduktion, die bis weit in die 1820er Jahre stagnierte ${ }^{53}$, stieg erst in den 1830ern allmählich an, ohne daß ein wirklicher Durchbruch, wie er dann nach $1850 \mathrm{zu}$ verzeichnen war, bereits damals gelang. Auch die Eisenhüttenindustrie zeichnete sich durch eine Kostenstruktur aus, in der mit 80-90 v. H. die Kosten für Rohund Brennstoffe den Löwenanteil verschlangen und die Lohnkosten der Hüttenarbeiter mit gerade 5 v. H. verschwindend gering waren. Fixkosten erwiesen sich auch hier, entgegen den Erwartungen, die man mit modernen Hüttenwerken verbindet, als eher gering.

\subsubsection{Stabeisenproduktion}

Die Stabeisenproduktion, d.h. die Weiterverarbeitung des im Hochofen gewonnenen Roheisens zu schmiedbarem Eisen, erfolgte in Oberschlesien bis weit ins 19. Jahrhundert hinein noch in den traditionellen Frischfeuern ${ }^{34}$, die mit Hammerwerken verbunden waren. Auch in diesem Industriezweig blieb der Einfluß des Adels zunächst überragend. Die Hauptstabeisenproduzenten waren der Graf Donnersmarck auf Beuthen-Siemianowitz sowie die Fürsten Hohenlohe/Slawentzitz und Ratibor. 1822 erzielten nicht-adelige Unternehmer einen Anteil von nur 2 v. H. an der gesamten Stabeisenproduktion. Die betreffenden Werke gehörten dem Ingenieur Wedding und der Landschaft, d.h. im letzteren Fall indirekt auch wiederum den Grundherren. Neben den Werken des Adels trat allein der preußische Fiskus mit seinen Werken als Anbieter von Stabeisen an den Markt ${ }^{55}$.

Erst nach 1833 gelang es einigen bürgerlichen Familien (Winckler, Adamietz, Heymann, Laband, Guradze), in diesem Produktionszweig Fuß zu fassen, und schließlich wies die Gründung der Aktiengesellschaft Minerva 1856 einen gänzlich neuen Weg zur Gestaltung der Eigentumsverhältnisse in der Eisenindustrie ${ }^{56}$. Zugleich fand die moderne Puddeltechnologie, die in Verbindung mit dem Eisenwalzen die traditionelle Stabeisenherstellung mittels Frischfeuer und Eisenhammer zunehmend ersetzte, auch in Oberschlesien weite Verbreitung: Zwar hatten bereits Ende der 1820er Jahre einige Eisenwerke begonnen, mit Puddelöfen zu experimentieren, doch dauerte es in Oberschlesien im Vergleich zu anderen deutschen Montanrevieren relativ lange, bis diese überlegene Technologie in die Stabeisenproduktion Eingang fand ${ }^{57} .1833$ nahm Fürst Hohenlohe in Althammer und im gleichen Jahr Graf Renard in Zandowitz ein Pud-

53 Vgl. die Produktionszahlen im Anhang S. 157.

54 Popiolek nennt sogar für 1881 noch die Zahl von neun in Betrieb befindlichen Frischfeuern; Górnoślạski przemyst, 102.

5s Kwaśny, Hutnictwo, 40, nennt mit Doms und Partnern erst für 1846 den ersten bürgerlichen Produzenten von Bedeutung, wenn man von Winckler absieht; ebenfalls dazu Kuaśny, Rozwój przemyslu, 45; Jerzy Jaros, Historia górnictwa węglowego w Zagłębiu Górnośląskim do roku 1914 [Geschichte des Kohlenbergbaus im oberschlesischen Revier bis 1914] (Breslau 1965) $274 \mathrm{ff}$.

56 Zur Minerva: Albert Hempelmann, Die Minerva, Schlesische Hütten-, Forst- und Bergbaugesellschaft. Ein Beitrag zur Entwicklung der oberschlesischen Industrie (Berlin 1936).

57 Kwaśny, Hutnictwo, 79, zählt für 1846 acht Puddelwerke in Oberschlesien, für 184970 Puddelöfen gegenüber 129 im Rheinland und 102 in Westfalen (ders., Rozwój, 71); den Stand zur Jahrhundertmitte resümiert Popiotek, Górnośląski przemysł, 29-31. 
delwerk in Betrieb. Zu diesem Schritt hatte sie die expandierende Nachfrage nach Stabeisen veranlaßt ${ }^{58}$, die sich dann in einer leicht und allmählich steigenden Produktion von Stabeisen in Oberschlesien niederschlug ${ }^{59}$.

\subsection{Zwischenbilanz}

Den ökonomischen „Erfolg“ der oberschlesischen Magnaten zu identifizieren ist vor dem Hintergrund der skizzierten Entwicklung der oberschlesischen Montanindustrie offenbar kein leichtes Unterfangen. Eine Differenzierung hinsichtlich des betrachteten Zeitraumes und des untersuchten Industriezweiges erscheint in jedem Fall angemessen.

Im alten, vormodernen Produktionssystem des 17. und 18. Jahrhunderts haben die Magnaten vermutlich ihre größten Erfolge erzielt. Ihre Vertreter waren an führender Stelle bei der damals neuen Methode der Eisenherstellung im Holzkohlenhochofen beteiligt; sie gründeten Eisenhütten zum Frischen des Roheisens und Hammerwerke zur Bearbeitung von Stabeisen. Insgesamt etablierten sie damit ein Produktionssystem, das nach dem damals geltenden Stand der Technik als äußerst modern zu bezeichnen ist. Aufgrund der Verfügung über grundherrlich gebundene Ressourcen konnten sie dieses Produktionssystem trotz geringer Produktivität und schlechter Qualität der Produkte auch profitabel nutzen. Damit trug diese vormoderne, vorkapitalistische Wirtschaftsweise zugleich bereits den Kern zu ihrem späteren Untergang in sich.

Denn sobald sich am Ende des 18. Jahrhunderts ein modernes, staatlich initiiertes Produktionssystem auch in der Montanindustrie Oberschlesiens etablieren konnte, geriet die alte Ökonomie in die Defensive. In der Übergangszeit zwischen 1800 und 1850 beherrschten die adeligen Grundherren zwar noch große Teile der industriellen Szene, in der Zinkindustrie bereits wesentlich weniger als im Kohlenbergbau und der Eisenindustrie, bürgerliche Konkurrenten traten aber hinzu. Diese bürgerliche Konkurrenz zwang die Magnaten bereits sehr früh zu Anpassungen und Veränderungen, und sie reagierten zunächst mit einer Aussonderung ihrer gewerblichen Unternehmen aus den feudalen Güterkomplexen und dem Versuch der Adaption kapitalistischer Wirtschaftsweisen in ihren industriellen Betrieben ${ }^{60}$. Dies gelang jedoch nur unvollkommen, weil weiterhin günstige Rohstoffe und Brennmaterialien, Halberzeugnisse, billige Arbeitskräfte sowie andere Vergünstigungen aus den Gutswirtschaften in Anspruch genommen wurden. Diese Ressourcen verschafften den Magnaten weiterhin einen Wettbewerbsvorteil und reduzierten den Druck zur Rationalisierung und Anpassung ihrer Betriebe. Ein eigentümliches Mischsystem mit Elementen der alten und der neuen Ökonomie konnte sich so lange halten, bis etwa zur Mitte des 19. Jahrhunderts das neue System seine unbestrittene Überlegenheit beweisen konnte. Dann

${ }^{38}$ Vgl. dazu Horst Wagenblass, Der Eisenbahnbau und das Wachstum der deutschen Eisen- und Maschinenbauindustrie 1835 bis 1860 (Stuttgart 1973).

59 Vgl. dazu die Produktionsdaten im Anhang S. 157.

${ }^{60}$ Dtugoborski, Die schlesischen Magnaten, 120. 
wurde eine umfassende Modernisierung der Betriebs- und Wirtschaftsstruktur nachweisbar: Die industriellen Besitztümer der Grundherren wurden zumeist bald als Aktiengesellschaften reorganisiert, und der eigentümliche Typus des oberschlesischen Magnaten verschwand mehr und mehr.

\section{Zum sozialen Umfeld der oberschlesischen Magnaten}

Daß sich die Unternehmer der oberschlesischen Montanindustrie insgesamt, nicht nur die Magnaten, sondern auch ihre bürgerlichen oder beamteten Konkurrenten, von der Unternehmerschaft etwa an der Ruhr deutlich unterschieden, wurde bereits an anderer Stelle aufgezeigt ${ }^{61}$. Für Oberschlesien gilt dabei festzuhalten, daß hier insbesondere der Einfluß des Staates und seiner Beamten sowie eben der Einfluß der bereits erörterten Magnaten bis zum Ende des 19. Jahrhunderts außergewöhnlich groß blieb. Einige besondere Merkmale der oberschlesischen Magnaten treten in dieser vergleichenden Betrachtung ebenfalls hervor. Ihr Eigentum an den Unternehmen der Schwerindustrie wurde zumeist noch in der rechtlichen Form eines Einzelunternehmers wahrgenommen, beschränkte sich jedoch zunehmend auf den Grundbesitz - während in der Eisenindustrie Kapitalgesellschaften an Boden gewannen - und reduzierte sich im Zeitverlauf insgesamt bedeutend.

Was die soziale Verortung dieser Unternehmerschaft angeht, so lassen sich ebenfalls einige charakteristische Merkmale der oberschlesischen Magnaten festhalten. Sie stammten überwiegend aus Familien, die seit langem in der Region ansässig waren. Zumeist verfügten diese über ausgedehnten Grundbesitz und widmeten sich dem Betrieb ihrer landwirtschaftlichen Güter. Technische oder kaufmännische Kenntnisse waren so gut wie nie vorhanden, eine formale Aus- und Vorbildung für eine Unternehmertätigkeit fehlte nahezu gänzlich. Sie schienen damit alles andere als gut vorbereitet für eine unternehmerische Karriere ${ }^{62}$.

Es wäre nötig, hinsichtlich der sozialen Struktur dieser Unternehmergruppe genauere Angaben zu mobilisieren. Dies gestaltet sich aber aufgrund der Quellenlage, die von der Überlieferung für einige „große “ Familien dominiert wird, in einer repräsentativen Weise als äußerst schwierig. Von insgesamt 90 Personen, die der Gruppe der oberschlesischen Magnaten zuzurechnen sind, läßt sich folgende Verteilung der Adelsprädikate bestimmen.

61 Toni Pierenkemper, Entrepreneurs in Heavy Industry: Upper Silesia and the Westphalian Ruhr Region, 1852 to 1913, in: Business History Review 1 (1979) 65-78.

62 Eine Ausnahme stellt Philipp Graf Colonna dar, der ,in Fragen der Eisenindustrie ... als erste Autorität [galt] und ... wiederholt in den Minister-Conseil bei Verhandlungen über volkswirtschaftliche und industrielle Gegenstände zugezogen [wurde]". (Matzner, Der letzte der schlesischen Colonna's, in: Breslauer Zeitung 1870, Nr. 163, 1266/67, u. 165, 1280/81, 1281); Franz Graf Ballestrem studierte zwar 1853-55 in Lüttich das Bergfach (vgl. Karl-Heinz Rother, Franz Graf von Ballestrem, in: Schlesische Lebensbilder Bd. 1 [1992] 247-251), überließ aber später die Leitung seiner Industrien Direktoren wie z. B. Franz Pieler. 


\begin{tabular}{|c|c|c|c|c|c|c|c|c|}
\hline \multirow[t]{2}{*}{ Adelsprädikat } & \multicolumn{2}{|c|}{$\begin{array}{l}\text { geboren } \\
\text { vor } 1800\end{array}$} & \multicolumn{2}{|c|}{$\begin{array}{l}\text { geboren } \\
1800 \text { und später }\end{array}$} & \multicolumn{2}{|c|}{$\begin{array}{l}\text { Geburtsdatum } \\
\text { unbestimmt }\end{array}$} & \multicolumn{2}{|c|}{ insgesamt } \\
\hline & $\mathbf{m}$ & $\mathbf{w}$ & m & $\mathbf{w}$ & $\mathrm{m}$ & $\mathbf{w}$ & $\mathrm{m}$ & w \\
\hline „von“ & 4 & - & 6 & - & 18 & 1 & 28 & 1 \\
\hline Baron/Freiherr & 2 & - & - & - & 2 & - & 4 & - \\
\hline Graf & 18 & 1 & 9 & 2 & 9 & 1 & 36 & 4 \\
\hline Fürst & 3 & - & 2 & - & 2 & 1 & 8 & 1 \\
\hline Herzog & 1 & - & 2 & - & 2 & - & 5 & - \\
\hline Prinz & 1 & - & - & - & 2 & - & 3 & - \\
\hline insgesamt & 29 & 1 & 19 & 2 & 35 & 3 & 84 & 6 \\
\hline
\end{tabular}

Man erfährt aus diesen wenigen Zahlen, daß eine Reihe hochrangiger Notablen zu den Magnaten zählten; immerhin acht Fürsten, sieben Herzöge und drei Prinzen, auch einige Frauen befanden sich darunter: Fürstin Sulkowska und die Gräfinnen Henckel, Schomberg-Godulla und Tiele-Winckler. Wenn auch die Zahl der Grafen, die in der oberschlesischen Montanindustrie beteiligt waren, mit 36 relativ groß erscheint, so reduziert sich deren Zahl in der praktischen Bedeutung auf wenige Namen, wie überhaupt die bedeutsamen Werke der Region von gut einer Handvoll Familien beherrscht wurden. Im wesentlichen handelt es sich dabei sogar nur um ganze sechs Familien, die seit dem frühen 19. Jahrhundert wesentliche Teile der oberschlesischen Montanindustrie kontrollierten, nämlich die beiden Linien Donnersmarck, Hohenlohe-Oehringen, Pleß, Schaffgotsch und Ballestrem. Einige frühe Pioniere hatten zu diesem Zeitpunkt bereits wieder an Einfluß verloren, so die Grafen Flemming, Hoym, Colonna und Renard; einige nobilitierte Bürgerfamilien, u.a. Winckler, Friedländer und Caro, traten später hinzu. Zur Veranschaulichung der sozialen Beziehungen innerhalb der hochadeligen Magnatengruppe sollen die sechs genannten „großen“ Familien Oberschlesiens kurz charakterisiert werden. Hinzu tritt ein Beispiel aus dem Kreis der frühen Pioniere und ein nobilitierter Bürgerlicher.

\section{Colonna/Renard}

Als einen der wichtigsten Pioniere der oberschlesischen Montanindustrie ist sicherlich Graf Philipp Colonna, Majoratsherr auf Groß-Strehlitz, zu nennen, der, geboren am 4. April 1755 auf der Burg Tost, auch gelegentlich als Initiator der oberschlesischen Eisenindustrie bezeichnet wird ${ }^{63}$. Schon mit 21 Jahren machte er, nachdem er in Göttingen und Halle Naturwissenschaften studiert hatte, 1776 eine Informationsreise zu den Eisenwerken im Harz, die seinerzeit als besonders fortschrittlich galten, und in das Siegerland, wo er auch mit dem Grafen Reden, dem späteren Förderer der Modernisierung der oberschlesischen Eisenindustrie, zusammengetroffen sein soll. Auch

${ }_{63}$ Perlick, Oberschlesische Berg- und Hüttenleute, 48. 
während seiner Studienreise nach England 1778-1779 galt neben der Landwirtschaft der Eisenindustrie sein Hauptinteresse ${ }^{64}$. Auf seinen ererbten Gütern betrieb Graf Colonna bereits zahlreiche Luppenfeuer, in denen er den Waldreichtum der Gegend und die leicht verfügbaren Eisenerze nutzte. 1782 wurde Colonna dann erstmals innovativ tätig, als er bei Groß-Stanisch, östlich von Oppeln, das Holzkohlehochofenwerk Colonnowska errichtete; diesem folgte bereits 1783 am linken Ufer der Malapane ein Stabeisenwerk mit Frischfeuern, das er nach seinem Güterdirektor Kowollik „Kowollowska“ benannte. 1780 hatte er bereits das Luppenfeuer Sandowitz, das 1752 durch seinen Vater begründet worden war, in ein Frischfeuer umgewandelt, und 1790 ergänzte er dieses ebenfalls mit einem Hochofen. 1792 entstand in Zborowski eine neue Hochofenanlage; 1795 erfolgte der Umbau des seit 1682 betriebenen Luppenfeuers in Neu-Zulkau in ein Frischfeuer mit Zainhammer, und schließlich wurde im gleichen Jahr auch das bereits seit 1530 bestehende Luppenfeuer Kotten durch einen Hochofen ersetzt ${ }^{65}$.

$\mathrm{Zu}$ Beginn des 19. Jahrhunderts (1804) verfügte Graf Colonna damit über Produktionsanlagen, bestehend aus drei Hochöfen, 15 Frischfeuern und zwei Zainhämmern mit einer Jahreskapazität von 20000 Ztr. Roh- und 16000 Ztr. Stabeisen. Ein bereits geplantes englisches Walzwerk konnte wegen der kriegerischen Verwicklungen PreuBens zu jener Zeit nicht gebaut werden. Diese weit über Oberschlesien hinaus bekannten Musterbetriebe ${ }^{66}$, deren Produkte wegen ihrer Qualität bis Stettin, Königsberg, 1791 sogar bis England Absatz fanden ${ }^{67}$, waren relativ modern, verfügten doch die Hochöfen bereits über ein Zylindergebläse, und zum Betrieb hatte der Graf eigens eine Schleuse an der Malapane errichten lassen, deren gespeichertes Wasser zum Antrieb eines eisernen Wasserrades benutzt wurde, welches wiederum Energie für die Gebläse und die Hämmer liefern konnte. Deshalb bemerkt Konrad Fuchs zu Recht, daß ,... die Bedeutung des Grafen ... weniger im Umfang seiner Produktionsstätten [liegt], sondern in den Anregungen, die von ihrem technischen Stand auf die weitere Entwicklung der oberschlesischen Industrie ausgingen“.

Graf Colonna blieb unverehelicht und ohne direkte Erben, so daß nach seinem Tod am 9. Juli 1807 der gesamte Besitz zunächst an den Baron von Gastheimb ging, der eine Colonna geheiratet hatte. Erst 1815 erfolgte eine endgültige Regelung der Erbschaft, und zwar dergestalt, daß Baron von Gastheimb die Hüttenwerke der Herrschaft Tworog, diejenigen im Bereich Groß-Strehlitz jedoch dem Grafen Andreas Maria Renard, dem Schwiegersohn von Gastheimbs, zufielen. Die Familie Renard stammte ursprünglich aus Frankreich, das sie jedoch um 1600 verlassen hatte, um sich in Hamburg im Kaufmannsgewerbe niederzulassen. Ein Mitglied der Familie, Andreas Renard, siedelte 1670 nach Warschau um, wo er als Handelsherr zu Ansehen und Reich-

${ }^{64}$ Gerbard Anter, Graf Philipp Colonna als Mitbegründer der oberschlesischen Eisenindustrie, in: Sonntagsbeilage der Schlesischen Zeitung, Nr. 47 (1924) 340.

${ }^{65}$ Joseph Kania, Die Reichsgrafen Colonna-Fels und die von üppiger Romantik reich umrankte Stammburg Tost, in: Schlesischer Musenalmanach 7 (1920) 107 f.; Perlick, Oberschlesische Bergund Hüttenleute, $48 \mathrm{f}$.

${ }^{66}$ Alfons Nowack, Philipp Graf Colonna, in: Schlesische Lebensbilder, Bd. 2 (1926) 119-122.

67 Anter, Graf Philipp Colonna, 341. 
tum gelangte. Er heiratete eine Gräfin Waldstein, und ihr Sohn Johann Baptist stieg als polnischer Offizier bis zum Generalleutnant auf und wurde in den Reichsgrafenstand erhoben. Dessen Sohn Johann Baptist II. heiratete eine Komteß Gaschin und gelangte so in den oberschlesischen Hochadel. Sie besaßen mehrere Güter in Preußen und Österreich und lebten in Wien, wo 1795 auch ihr Sohn Andreas Maria geboren wurde.

Andreas Maria Renard erbte nun nicht nur den reichen väterlichen Besitz, sondern darüber hinaus 1815, also mit zwanzig Jahren, über seinen Schwiegervater Baron von Gastheimb aus der Hinterlassenschaft des Grafen Colonna noch die Herrschaft Groß Strehlitz mit den dort bestehenden modernen Industrieanlagen aus der Hinterlassenschaft des Grafen Colonna. Diese fanden zweifellos großes Interesse bei dem jungen Grafen, und er widmete sich mit Eifer dem Ausbau der Anlagen ${ }^{68} .1819$ begann er links und rechts der Malapane mit dem Bau einer neuen Hütte, der Renardhütte mit Frischfeuern, einem Blechwalzwerk und einem Blechhammer. 1821 konnten dort vier Frischfeuer in Betrieb gesetzt werden. 1824 erweiterte er das Hochofenwerk Colonnowska um einen weiteren Holzkohlehochofen, hinzu kam ein Drahtwerk und eine mechanische Werkstätte. 1830-32 modernisierte Renard die Anlagen in Sandowitz und errichtete dort ein modernes Puddel- und Walzwerk, und auch das Zawadzkiwerk, das 1836 ursprünglich als Frischfeuerhütte mit Reckhämmern entstanden war, wurde 1841 zum Puddel- und Walzwerk umgebaut. 1843 ging dieses in Betrieb und wurde bereits 1844 um ein Tiegelstahlwerk ergänzt.

1851 übernahm Renard auch die bereits 1840 gegründete Friedenshütte bei Beuthen, auf der ein Kokshochofen bestand. Erst sehr spät vollzog damit der Graf bei der Roheisengewinnung den Schritt zur modernen, richtungweisenden Steinkohlentechnologie und die Verlagerung der Produktion ins Kohlenrevier. Dieses Werk wurde nun auch modernisiert, u. a. durch einen neuen Hochofen mit vergrößertem Fassungsvermögen und einer Hochdruck-Gebläse-Dampfmaschine; zugleich wurden auch eigene Koksöfen zur Herstellung des Koks errichtet. Zur Mitte des Jahrhunderts (1853) verfügte Graf Renard auf dem Höhepunkt seiner unternehmerischen Aktivitäten über insgesamt 22 kleinere und größere Eisenwerke sowie über Steinkohlengruben mit ausgezeichneter Kokskohle in der Nähe von Beuthen. Die Jahresproduktion der Anlagen lag bei 150000 Ztr. Roheisen (10 v. H. der Gesamtproduktion Oberschlesiens), 130000 Ztr. Stabeisen (25 v. H.) und 15000 Ztr. Eisenblech (50 v. H.). Dennoch zog er sich kurz danach aus dem aktiven Unternehmerleben zurück. Einige schwere Schicksalsschläge mögen dazu beigetragen haben: 1854 verstarb seine Frau, $1855 \mathrm{kam}$ sein jüngster Sohn bei einem Jagdunfall zu Tode.

1855 brachte Graf Andreas Maria Renard sein gesamtes Vermögen zum Kaufpreis von knapp 3,5 Mill. Talern in die „Schlesische Hütten-, Forst- und Bergbaugesellschaft Minerva" ein, in deren Aufsichtsrat er den Vorsitz übernahm ${ }^{69}$, und zog sich bis zu seinem Tode 1875 aus dem aktiven Geschäftsleben zurück. Sein Sohn Johannes Graf

${ }^{68}$ Zu Renard: Vgl. Anm. 50, Fucbs; Zbigniew Pustuta, Renard, in: Polski Stownik Biograficzny [Polnisches Biographisches Wörterbuch] Bd. 31 (1988) $103 \mathrm{f}$.

69 Zur Minerva s. Anm. 56; Hempelmann gibt eine sehr kritische Darstellung des Unternehmers Renard. 
Renard war von 1868 bis zu seinem frühen Tod 1874 Vorsitzender des Verwaltungsrates der Oberschlesischen Eisenbahn-Bedarfs AG, dem Nachfolgeunternehmen der Minerva.

\section{Hohenlohe-Oehringen (Flemming/Hoym)}

Noch früher als der Graf Colonna wurde der sächsische Minister Jakob Heinrich Graf Flemming in der oberschlesischen Eisenindustrie aktiv. Dieser erwarb 1702 die Herrschaft Slawentzitz von der verwitweten Gräfin Juliana Henckel von Donnersmarck und errichtete dort 1703, nahe Kieferstädtel, einen Holzkohlehochofen, der als erster Hochofen Oberschlesiens überhaupt gilt. 1709 folgte die Gründung des Eisenwerkes Blechhammer. 1714 gelangte die Herrschaft Ehrenforst in den Besitz des Grafen Adolf Magnus Hoym, der die bestehenden Industrieanlagen weiter ausbaute. Hoym starb 1723 kinderlos, und das Erbe fiel seinem Neffen Julius Gebhardt Hoym zu, der bereits über eigenen Industriebesitz verfügte und 1751 aus der Kokursmasse des Pächters und Verwalters des Hoymschen Besitzes, Georg von Jänisch, der eigene Anlagen erworben hatte, noch zwei Hochöfen in Althammer und Goschütz sowie eine Reihe von Frischfeuern erwarb ${ }^{70} .1769$ verstarb der Graf und hinterließ seiner Tochter Amalie Marianne Gräfin Hoym einen beachtlichen Industrie- und Güterbesitz, der durch ihre Heirat mit dem Erbprinzen Hohenlohe-Ingelfingen 1782 in den Besitz der Familie Hohenlohe gelangte und zur Basis ihres montanindustriellen Vermögens in Oberschlesien wurde ${ }^{71}$.

Ab 1801 erfolgten durch den Fürsten Hohenlohe-Ingelfingen weitere Erwerbungen in Oberschlesien: Das 1801 erworbene Rittergut Bittkow erwies sich als besonders ertragreich $^{72}$, weil dort bereits eine Zinkhütte bestand, zu deren Versorgung eine Galmeigrube (Hohenlohegrube) wieder in Betrieb genommen wurde. 1804 wurde dort ein Hochofen angeblasen, gleichzeitig wurden weitere Erwerbungen andernorts gemacht, so die Herrschaften Koschentin, Tworog, Landsberg und Czechowa.

1818 verstarb der Fürst, und sein Sohn Friedrich August Karl von Hohenlohe-Oehringen setzte das Werk seines Vaters fort. Den Schwerpunkt der industriellen Tätigkeit bildete zunächst noch die Zinkindustrie: 1841 wurde die Scharley Grube, 1847 die Gruben Neue Helene und Brzozowitz gemutet. Hugo Fürst zu Hohenlohe-Oehringen (1816-1897), seit 1861 Herzog von Ujest und 1871-1875 Vizepräsident des Reichstags, folgte seinem Vater 1849 in der Verwaltung des Familienbesitzes nach und baute die Zinkindustrie bedeutend aus. 1871 errichtete er die Hohenlohe-Zinkhütte in Bittkow, 1888 kam ein Zinkwalzwerk dazu. Nach dem Ankauf der Theresien-

${ }^{70} \mathrm{Zu}$ Flemming und Hoym: Oswald Volkel, Geschichte der Industrie im Gleiwitzer Raum, in: Der Oberschlesier 21 (1939) 442 f.; Paul Klein, Alte Industrien in der Bischofstaler Gegend, in: Der Oberschlesier 20 (1938) 695-699.

${ }^{71}$ Bruno Knochenbauer, Die oberschlesische Montanindustrie (Gotha 1927) 116-120; Kurt Repetzki, Geschichte der oberschlesischen Montanindustrie, 9. Hohenlohe-Oehringen, in: Gleiwitzer und Beuthener Heimatblatt 1 (1952) Nr. 11 u. 19.

${ }^{72} \mathrm{Vgl}$. Perlick, Oberschlesische Berg- und Hüttenleute, 46; Piernikarczyk, Historia górnictwa, Bd. 2, 162-164. 
Zinkhütte in Michalkowitz (1892) und der Pachtung der Godulla-Hütte der Gräfin Schaffengotsch (1895) galt Hugo Fürst Hohenlohe in den 90er Jahren als der größte Zinkproduzent der Welt ${ }^{73}$. Mit über 41587 ha war er auch der größte Grundbesitzer in Oberschlesien ${ }^{74}$. Neben der Zinkindustrie wurden nun auch Steinkohlengruben (ab 1869) erworben und die Eisenhütte (ab 1871) ausgebaut. Diese Richtung verfolgte auch Christian Kraft Fürst von Hohenlohe-Oehringen weiter, bis schließlich 1905 der Großteil des Hohenloheschen Industrievermögens in eine Aktiengesellschaft eingebracht wurde. Dafür erhielt Christian Kraft eine einmalige Abfindung von 44 Mill. Mark sowie eine jährliche Rente von 3 Mill. Mark. Die Abfindung verwandte der Fürst zum Aufbau eines großen Konzerns, der 1913 in einen spektakulären Zusammenbruch geriet, in den auch die Hohenlohe AG mit hineingezogen wurde ${ }^{75}$.

\section{Ballestrem}

Carl Franz Graf von Ballestrem, Sohn des Giovanni Baptista Angelo Balestreri die Castellengo, eines savoyischen Offiziers in preußischen Diensten, und der Elisabeth Auguste Freiin von Stechow, hatte bis 1797 im oberschlesischen Kürassierregiment Nr. 12 in Ratibor zuletzt als Major in Dienst gestanden ${ }^{76} .1798$ gelangte er durch eine Erbschaft in den Besitz des Stechowschen Majorats und hatte dort zunächst große Schwierigkeiten wegen der strittigen Besitzverhältnisse an den dortigen Steinkohlegruben Brandenburg und Maximiliane. Nachdem diese geklärt waren, konzentrierte sich Ballestrem zunächst auf den Bergbau und legte in den folgenden Jahren zahlreiche Mutungen ein, u.a. 1808 Bessere Zukunft und Johannessegen, 1810 Gute Schifffahrt. Anfänglich wegen der erforderlichen Investitionen vor der Errichtung von zwar rentablen, aber auch konjunkturanfälligen Hütten zurückschreckend, entschloß er sich 1812 auf die Initiative seines dynamischen Verwalters Godulla hin zum Bau der CarlsZinkhütte in Ruda, einer der "größten und technisch perfektesten Anlagen " ${ }^{\text {"77 }}$ dieser Art. Von kurzen Zeiten abgesehen, in denen die Ballestrems diese vorbildliche Zinkhütte auf eigene Rechnung betrieben, war sie seit 1855 an die Schlesische AG für

${ }^{3}$ Alfons Perlick, Hugo Fürst zu Hohenlohe-Oehringen, NDB, Bd. 9, 492; Popiotek, Górnośląski przemyst, 159,164 f., $172-174$.

74 Stefan Migdal, Z badań nad ekonomiczno-spolecznym podłożem kwestii narodowej na Górnym Śląsku w początku XX wieku [Aus der Forschung zum wirtschaftlich-sozialen Hintergrund der nationalen Frage in Oberschlesien zu Anfang des 20. Jahrhunderts] (Oppeln 1966) 20.

75 Einen guten Überblick über die Entwicklung der Hohenloheschen Unternehmungen bis zur Bildung der „Hohenlohe-Werke AG zu Hohenlohe-Hütte O/S“ gibt Lech Szaraniec, Zatoga koncernu „Hohenlohe“ i jej walka klasowa w latach 1905-1939 [Die Belegschaft des „Hohenlohe“Konzerns und ihr Klassenkampf in den Jahren 1905-1939] (Kattowitz 1936) 13-43.

${ }^{76} \mathrm{Zu}$ den Freiherrn von Stechow: Knescbke, Neues allg. dt. Adelslexikon, Bd. VIII (1929); Ballestrem: Ernst Laslowski, Zur Geschichte der Grafen von Ballestrem, in: Schlesien 77 (1943) 8894; Jaros, Tajemnice, $15 \mathrm{f}$.

${ }_{77}$ Fucbs, Zur Bedeutung des schlesischen Magnatentums für die wirtschaftliche Entwicklung Oberschlesiens, in: Beiträge zur Wirtschafts- u. Sozialgeschichte, 127. 
Bergbau und Zinkhüttenbetrieb, später an die Hohenlohe-Werke verpachtet ${ }^{78}$. Nach dem Tod des Grafen Carl Franz 1822 widmete sich dessen Bruder Carl Ludwig dem weiteren Ausbau der Anlagen - er nahm u.a. 1823 den ersten großen Tiefbau auf der Brandenburggrube in Angriff ${ }^{79}$-, bis auch er 1829 verschied.

Bereits 1808 war jedoch Karl Godulla, im 27. Lebensjahr stehend, zum Generalbevollmächtigten der Ballestremschen Güter berufen worden. Während des Zinkbooms in den 1820er Jahren mutete er auf eigene Rechnung eine Reihe von Galmeigruben und setzte damit den Grundstein zu einem beachtlichen industriellen Vermögen. 1830 löste er daher sein Dienstverhältnis mit dem Grafen Ballestrem wieder auf, blieb den Ballestremschen Industrieinteressen jedoch weiterhin verbunden. Ab 1829 war nun auch Carl Wolfgang Graf Ballestrem in die Leitung des Familienvermögens eingetreten und betrieb zwischen 1841 und 1857 den Ankauf zahlreicher Galmei- und Steinkohlengruben. 1854 verkaufte er $\mathbf{u}$.a. auch mehrere Gruben und ein Grundstück an August Borsig zur Anlage seines Eisenhüttenwerkes in Oberschlesien. Sein Sohn Franz, der mit klarem Blick für die Bedeutung der Steinkohle für Oberschlesien das Bergfach studiert hatte, übernahm 1879 die Familienbesitzungen; zwar widmete er sich als Reichstagsmitglied (1872-1906) und Reichstagspräsident (1889-1906) mehr der großen Politik. Mit Franz Pieler engagierte er jedoch 1885 einen zielbewußten Leiter, während dessen fünfundzwanzigjähriger Tätigkeit für die Ballestremschen Werke diese ohne Inanspruchnahme fremden Kapitals bedeutend erweitert wurden (u. a. 1895 Castellengo-Grube), so daß sich die Förderung bis 1912 mehr als versechsfachte ${ }^{80}$. Anfang des 20. Jahrhunderts nahm Ballestrem mit einem Anteil von $6,6 \%$ an der oberschlesischen Förderung den fünften Platz unter den oberschlesischen Kohlenmagnaten $\operatorname{ein}^{81}$.

\section{Schaffgotsch/Godulla}

Karl Godulla (1781-1848), Sohn eines Waldaufsehers, der entgegen der Legende ein nicht unbeträchtliches Vermögen besaß und dessen Tochter den reichen Gleiwitzer Baron August von Lengsfeld heiratete ${ }^{82}$, trat nach Beendigung der Mittelschule in die Dienste des Grafen Carl Franz Ballestrem und gelangte schnell in die Stellung eines Verwalters $^{83}$. Godulla bewog Ballestrem zur Errichtung der Carls-Zinkhütte (1812), des ersten modernen Zinkhüttengroßbetriebes in Oberschlesien ${ }^{84}$. Die Godulla von Ballestrem geschenkten $28 \mathrm{Kuxe}$ an diesem bald zur größten Zinkhütte Europas avan-

\footnotetext{
78 Vgl. Jaros, Tajemnice, 16 f.; Dworak, Karol Godula, 51-54; Perlick, Oberschlesische, $51 \mathrm{f}$.

79 Laslowski, Zur Geschichte, 92.

${ }^{80}$ Vgl. Knochenhauer, Die oberschlesische Montanindustrie, 120-122; zu Franz Graf Ballestrem: Helmut Neubach, Franz Graf Ballestrem, ein Reichstagspräsident aus Oberschlesien (Dülmen 1984); Herbert Ebren, Graf Franz Ballestrem. Ein Lebensbild (Breslau 1935); zu Franz Pieler: Perlick, Oberschlesische Berg- u. Hüttenleute, $166 \mathrm{f}$.

${ }^{81}$ Popiotek, Górnośląski przemysł, 137.

${ }^{82}$ Vgl. Emil Drobny, Rodzina Godulów [Die Familie der Godula], in: Zaranie Śląskie [Schlesische Morgenröte], 11 (1935) 179-181.

${ }^{83}$ Dworak, Karol Godula, $48 \mathrm{f}$.

${ }^{84}$ Perlick, Oberschlesische Berg- und Hüttenleute, 52.
} 
cierenden Werk wurden zum Grundstock für dessen eigene industriellen Unternehmungen $^{85}$ : Zwischen 1836 und 1847 erwarb bzw. errichtete Godulla die Zinkhütten Gutehoffnung, Bobrek und Morgenroth; mit 40300 Ztr. hatte er einen Anteil von $10,61 \%$ an der jährlichen Zinkproduktion in Oberschlesien ${ }^{86}$. Als Karl Godulla, der bis 1830 im offiziellen Dienst des Grafen Ballestrem stand, 1848 an der Cholera starb, besaß er 4 Zinkhütten, 18 Galmeischächte, 40 Kohlengruben: Sein Vermögen wurde auf 2 Millionen Taler geschätzt ${ }^{87}$. Dieses enorme Vermögen vererbte er Johanna Gryzik, der Tochter eines Bergmannes, die er in vorgerücktem Alter an Kindes Statt angenommen und erzogen hatte. 1858 wurde sie unter dem Namen von Schomberg-Godulla geadelt und vermählte sich im gleichen Jahr mit dem Grafen Hans Ulrich Schaffgotsch. Damit war der Grundstock zum Schaffgotschen Industrievermögen gelegt, das sich bis 1913 vor allem auf Steinkohlengruben stützte $(6,3 \%$ der oberschlesischen Förderung) ${ }^{88}$, nachdem die Gräfin Schaffgotsch durch Verkauf oder Verpachtung ihrer Hütten in den 90er Jahren ihr Kapital aus der Zinkindustrie zurückgezogen hatte $^{89} .1905$ wurde der Schaffgotsche Besitz in die Gräflich Schaffgotsche Werke $\mathrm{mbH}$ mit einem Grundkapital von 50 Mill. Mark eingebracht; das von Karl Godulla geerbte Vermögen war demnach innerhalb eines halben Jahrhunderts achtmal größer geworden $^{90}$.

\section{Pleß}

Die Standesherrschaft Pleß existierte seit 1447 und war 1765 an den Prinzen Friedrich Erdmann von Anhalt-Köthen gekommen. Seit 1754 wurde dort auf der Grube Emanuelssegen bereits Steinkohle gefördert, so daß diese Grube als älteste des oberschlesischen Reviers gelten kann. 1774 wurde die Förderung jedoch wieder eingestellt, weil billigere Kohle aus dem Waldenburger Revier zu beziehen war ${ }^{91} .1847$ gelangte die Standesherrschaft Pleß in den Besitz des Grafen Hans Heinrich X., Reichsgraf von Hochberg, Freiherr von Fürstenstein, der ein Neffe des letzten Herzogs von AnhaltKöthen war. Zwischen 1846 und 1855 investierte der erste Hochberg, dem 1850 die erloschene Fürsteneigenschaft wiederverliehen worden war $^{92}$, in den vernachlässigten industriellen Besitz; Hans Heinrich XI. setzte die Aufbauarbeit seines Vaters fort, wobei sein Interesse ,in weiser Berücksichtigung der geographischen Lage dieses seines Besitzes, der mannigfachen ... wirtschaftlichen Momente" vor allem der Landwirtschaft galt: In den 60er und 70er Jahren mußten die auf Holzkohle basierenden Eisenhütten in Paprotzan und Panewnik, die Idahütte bei Ellgoth und die Adelheidhütte

Bs Dworak, Karol Godula, $53 \mathrm{ff}$.

${ }^{86}$ Dworak, Karol Godula, 59.

${ }^{87}$ Klara Marysówna-Meixnerowa, Karol Godula, in: Polski Slownik Biograficzny, Bd. 8 (Warschau 1959/60) 192.

${ }^{88}$ Popiotek, Górnośląski przemysil, 137.

${ }^{89}$ Jaros, Tajemnice, 135.

90 Ebd. $137 \mathrm{f}$.

91 Ezecbiel Zivier, Die Entwicklung des Steinkohlenbergbaus im Fürstentum Pleß (Kattowitz 1913) 19-39.

92 Ebd. 14. 
bei Gostin geschlossen werden ${ }^{93}$. Im Steinkohlenbergbau dagegen kam es unter Hans Heinrich XI. zu einer beschleunigten Entwicklung, so daß die Förderung sämtlicher Plesser Gruben von 400000 t p.a. in 1855 nach Eröffnung mehrerer neuer Anlagen 1906 bei $664000 \mathrm{t} \mathrm{lag}^{94}$.

\section{Donnersmarck}

Die Familie Donnersmarck stammte aus Ungarn. Lazarus Henckel von Donnersmarck (1551-1624) erhielt 1623 die Herrschaft Beuthen als Pfand von Kaiser Ferdinand II., und da dieses Pfand nicht eingelöst wurde, ging die Herrschaft 1836 in den erblichen Besitz der Familie. 1670 kam es zu einer Teilung des Besitzes in eine katholische Beuthener und eine protestantische Neudecker Linie ${ }^{95}$.

Zunächst war die Beuthener Linie hinsichtlich der industriellen Aktivität der Neudecker Linie deutlich voraus. Diese begründete bereits 1806 die Antonienhütte, das erste private Kokshochofenwerk in Oberschlesien, und widmete sich unter Lazarus III. (1729-1805) dem Ausbau des Steinkohlenbergbaus (1787 Glücksgrube, 1802 Gottessegen, die bis Anfang des 20. Jahrhunderts zu den ertragreichsten Gruben zählte) ${ }^{96}$. Graf Hugo I. Henckel von Donnersmarck, sein Enkel, baute die Antonienhütte weiter aus. 1836 nahm er seine bedeutendste industrielle Gründung, die Laurahütte, in Angriff, das erste und größte gemischte Werk in diesem Revier mit Gewinnung des Rohmaterials bis zur Produktion von Walzwerkprodukten. 1869, im Zenit seiner industriellen Laufbahn, kaufte Hugo Henckel die fiskalische Königshütte; seine Werke lieferten in diesem Jahr 90\% der oberschlesischen Walzwerk- und 49\% der Roheisenproduktion; seine Eisenerzgruben erbrachten 32\%, die Kohlengruben 13,3\% der oberschlesischen Gesamtförderung; an der oberschlesischen Zinkproduktion war er mit $17 \%$ beteiligt $^{97}$.

Königs- und Laurahütte wurden 1871 mit den dazugehörigen Steinkohlengruben und einigen Eisenerzfeldern an die „Vereinigte Königs- und Laurahütte AG“ verkauft. Die Aktien der „Königslaura“, deren Mehrheit Hugo Henckel hielt, waren eines der begehrtesten Papiere an der Berliner Börse ${ }^{98}$. Eine Reihe von Kohlengruben wurden weiterhin von der Gräflich Donnersmarckschen Güterverwaltung betrieben.

Die Neudecker Linie verstärkte ihr industrielles Engagement erst, nachdem 1848 Graf Guido Henckel von Donnersmarck die Erbfolge angetreten hatte. Bis dahin war dort hauptsächlich Land- und Forstwirtschaft betrieben worden; ansonsten existierten lediglich der Hochofen Brinitz sowie einige Eisen- und Galmeigruben. Planvoll wid-

${ }^{93}$ Ezecbiel Zivier, Hans Heinrich XI., Herzog von Pleß, in: Oberschlesien 6, H. 7 (1907) 309318.

${ }^{94}$ Ebd. 316.

${ }^{95}$ August Dörr, Beiträge zur Geschichte und Genealogie der Familie Henckel von Donnersmarck (1908); Josepb Kania, Schloß Neudeck und das Geschlecht der Grafen Henckel von Donnersmarck, in: Schlesien $1(1907 / 08) 493 \mathrm{f}$.

96 Czaja, Der Aufstieg, 29-31.

97 Ebd. 34ff., 42.

${ }_{98}$ Popiotek, Górnośląski przemysł, 79. 
mete sich dann der Graf dem Auf- und Ausbau seiner schwerindustriellen Interessen $^{99}$. 1853 gründete er unter finanzieller Beteiligung des größten festländischen Zinkproduzenten, der belgischen Vieille Montagne, die „Schlesische Aktiengesellschaft für Bergbau und Zinkhüttenbetrieb“, deren Aufsichtsratsvorsitzender er 63 Jahre lang blieb ${ }^{100}$, ebenso die Donnersmarck-Hütte, die 1872 in eine AG umgewandelt wurde und in deren Aufsichtsrat er bis 1885 Mitglied war ${ }^{101}$. Die erheblich modernisierte Falvahütte wurde 1908 an die Bismarckhütte verkauft. Guido Henckel, der mit einer Roheisenerzeugung von 1800 t p.a. begonnen und dessen Werke 1916 über $500000 \mathrm{t}$ produzierten ${ }^{102}$, konzentrierte sich mit sicherem Gespür für die begrenzten Chancen der oberschlesischen Eisenindustrie zunehmend auf den Kohlenbergbau. Die Förderung der Henckelschen Gruben wies eine kontinuierliche Steigerung auf $(1863=17487 \mathrm{t} ; 1913=2370639 \mathrm{t})^{103}$; er nahm damit den achten Platz unter den oberschlesischen Kohlenproduzenten ein. Seit den 90er Jahren wandte er sich verstärkt der Zinkindustrie zu (u.a. 1887 Bau der Guidotto-Hütte bei Chropaczow) ${ }^{104}$. Guido Graf Henckel, der neben Krupp, Thyssen, Rathenau u.a. zu den Begründern der deutschen Großindustrie zählt, betätigte sich auch in der chemischen und in der Baustoffindustrie ${ }^{105}$. Die oft kühnen Unternehmungen des 1901 gefürsteten Guido Henckel von Donnersmarck standen stets auf finanziell solidem Fundament - im Gegensatz zum Finanzdilettantismus einiger seiner Standeskollegen ${ }^{106}$. In den höchsten politischen Kreisen Berlins wurde der Rat Henckels, seit 1887 erbliches Mitglied des preußischen Herrenhauses, der über Aufstieg und Fall von Ministern mitentschieden haben soll, geschätzt ${ }^{107}$.

\section{Tiele-Winckler}

Franz Winckler wurde 1803 in Tarnau bei Frankenstein geboren und kam 1818 nach Oberschlesien, wo er auf einem Erzbergwerk im Tarnowitzer Revier als Bergmann tätig wurde. Wegen seiner offensichtlichen Talente wurde er dem königlichen Bergamt zur

${ }^{99} \mathrm{Zu}$ Guido Henckel von Donnersmarck: Czaja, Der Aufstieg; Ulrich Lobse, Guido Graf Henckel von Donnersmarck und seine industriellen Schöpfungen, in: Stahl und Eisen 37 (1917) 156-161; Arthur Friedrich, Oberschlesische Industriekapitäne, in: Nord und Süd 40 (1916) 102104; Konrad Fuchs, Guido Georg Friedrich Graf Henckel von Donnersmarck (1830-1916), in: Ostmitteleuropa. Festschrift für Gotthold Rhode (Stuttgart 1981) 237-251.

${ }_{100}$ Lobse, Guido Graf, 153; Fuchs, Guido Georg, 238.

${ }^{101}$ Albert Hempelmann, Die Donnersmarckhütte AG, ein Stück oberschlesischer Industriegeschichte, in: Oberschlesische Wirtschaft 6, H. 1 (1931) 19-24; H. 2, 97-105, bes. 98.

102 Lobse, Guido Graf, 159.

103 Czaja, Der Aufstieg, 71.

104 Ebd. 76; Popiotek, Górnośląski przemyst, 173.

105 Lobse, Guido Graf, 157, 160.

106 Felix Pinner, Deutsche Wirtschaftsführer (Berlin 1925) 84.

107 Ludwig Stein, Bismarck und Fürst Guido Henckel von Donnersmarck, in: Nord und Süd 39 (1915) 261-266; Helmut Böbme, Guido Graf Henckel von Donnersmarck, Bismarck und der Krieg von 1866, in: Tradition. Zeitschrift für Unternehmensgeschichte 12 (1967) 378-387; Bernbard Fürst von Bülow, Denkwürdigkeiten, Bd. 4 (Berlin 1929) 493; Rudolf Martin, Deutsche Machthaber (Berlin 1910) 225-231, bes. $235 \mathrm{f}$. 
weiteren Ausbildung empfohlen und besuchte seit 1821 die Bergschule in Tarnowitz, die jedoch nach dem Tode des dortigen Leiters Stroh wieder aufgelöst wurde. Statt dessen nahm er nach dem kurzen Besuch der Bergschule eine Schichtmeisterstelle auf der Mariagrube bei Miechowitz an und wurde nach Bewährung dort von dem Besitzer, dem Freiherrn von Aresin, zum Bergwerksleiter gemacht. Aresin verstarb bald darauf, und Franz Winckler machte sich durch seine Tätigkeit gänzlich unentbehrlich. 1829 verstarb seine Frau Alvine Kalide, so daß er 1832 mit seiner Dienstherrin Maria Aresin eine neue Ehe eingehen konnte und so auch in den Besitz der von ihm bereits verwalteten Betriebe kam. Franz Winckler widmete sich dann verstärkt dem Ausbau seiner Industrieunternehmen, unterstützt u. a. durch eine Studienreise nach England, und wurde wegen seiner Verdienste für die oberschlesische Industrie 1840 geadelt ${ }^{108}$.

Franz und Maria Winckler hatten sich in mehreren Prozessen die Zuerkennung der Regalrechte auf den 1838 und 1839 erworbenen Rittergütern Kattowitz und Myslowitz unter Berufung auf deren ehemalige Zugehörigkeit zur Standesherrschaft Pleß erstritten ${ }^{109}$. Mit seinem umfangreichen Landbesitz, der außerdem noch 6 weitere Güter nebst zugehörigem, zielstrebig weiterentwickeltem Industriebesitz und reichen Steinkohlengruben umfaßte ${ }^{110}$, zählte Winckler neben den Hohenlohe, Henckel von Donnersmarck, Schaffgotsch, Ballestrem zum Kreis der bedeutendsten oberschlesischen Magnaten. Die eigenen Eisen-, Brauneisenerz- und Toneisenerzsteinlager deckten bis in die 60er Jahre den Bedarf der Winckler/Tiele-Winckler'schen Eisenhütten (1836 Dietrichshütte, 1837 Valeskahütte und Doppelfrischfeuer bei Jeschonka, 1838 Mariahütte und Sophienhütte) ${ }^{111}$.

Nach seinem Tode 1851 wurden seine Unternehmen, die sich nun auch von den Erzgruben hin zur Eisenverhüttung und Eisenverarbeitung entwickelt hatten, von seinem langjährigen Freund und Mitarbeiter Grundmann ${ }^{112}$ verwaltet. Das änderte sich auch nicht, als nach dem Tode seiner zweiten Frau die Tochter aus erster Ehe, Valeska von Winckler, das bedeutende Erbe antrat. Auch nach deren Heirat mit dem Leutnant von Tiele, der sich dann von Tiele-Winckler nannte, blieb Grundmann der Generalverwalter des Herrschaftsbesitzes, der sich durch den Erwerb der Herrschaften Kattowitz (1838) und Myslowitz (1839) entscheidend ausgedehnt hatte.

${ }^{108}$ Zu Franz Winckler: Ludwig Chrobok, Beiträge zur Lebensgeschichte Franz von Wincklers, in: Mitteilungen des Beuthener Geschichts- und Museums-Vereins, H. 5 (1924) 14-18; Joseph Kania, Das historische Dreigestirn am Werdehimmel der Großindustrie Oberschlesiens; Georg von Giesche, Karl Godulla, Franz von Winckler, in: Schlesischer Musenalmanach 6 (1920) 179194; Piernikarczyk, Historia górnictwa, Bd. 2, 228-236; Konrad Fuchs, Franz von Winckler, in: Schlesische Lebensbilder, Bd. V (Würzburg 1968) 107-112.

109 Jerzy Jaros, Dzieje przemyslu górniczo-hutniczego na terenie Mystowic [Geschichte der Bergund Hüttenindustrie im Myslowitzer Gebiet], in: Wactaw Dlugoborski u. a. (red.), Másłowice (Kattowitz 1977) 38; Fuchs, Vom Dirigismus, 207.

"10 Konrad Fuchs, Ursprung und Entwicklung der Industriestadt Kattowitz, in: Jahrbuch der Schlesischen Friedrich Wilhelm Universität XXVII (1986) 152, nennt 14 Galmei- und 69 Steinkohlengruben (ganz oder anteilmäßig), 7 Zinkhütten mit einer Jahresproduktion von 110000 Ztr. Zink, 6 Hochöfen mit 100000 Ztr. Roheisen p.a., ein Walzwerk und zwei Frischfeuer mit einer Kapazität von $40000 \mathrm{Ztr}$. Schmiedeeisen.

11 Grundmann, Friedrich Wilhelm Grundmann, 130-131.

112 Zu Friedrich W. Grundmann: vgl. Anm. 41. 
1880 starb Valeska von Tiele-Winckler. Ihr Mann lebte bis 1893, und ihr gemeinsamer Sohn Graf Franz-Hubert von Tiele-Winckler, geb. 1853, trat ein Erbe-an, das schon 1889 in der Kattowitzer AG für Bergbau und Eisenhüttenbetrieb mit TieleWinckler als Hauptaktionär zusammengefaßt worden war und lange Jahre das führende Industrieunternehmen Oberschlesiens darstellte ${ }^{113}$.

\section{Ausblick}

Bis zur Mitte des 19. Jahrhunderts waren die oberschlesischen Magnaten zumindest teilweise und mit unterschiedlichem Erfolg aktiv in der Unternehmenspolitik der Schwerindustrie beteiligt. Ab etwa 1850, als in Deutschland insgesamt der Durchbruch zur Industrialisierung gelang und erst dann die Montanindustrie zum Führungssektor wurde, lassen sich für Oberschlesien zwei entscheidende Entwicklungen festhalten: Zum einen erfolgte eine deutliche Konzentration der Eigentumsverhältnisse ${ }^{114}$ in der Montanindustrie, und zum anderen verlor diese Region ihre Führungsrolle innerhalb der deutschen Montanbezirke ${ }^{115}$.

Bei der Konzentration der Eigentumsverhältnisse waren die großen Magnatenfamilien entscheidend beteiligt, und sie vermochten so ihren Einfluß auf die oberschlesische Industrie sogar noch zu steigern. Die acht großen Familien und der preußische Fiskus betrieben 185654 von 91 fördernden Steinkohlengruben, produzierten damit etwa 80 v. H. der Gesamtförderung des Bezirks und beschäftigten etwa 77 v. H. aller Bergleute ${ }^{16}$. Ähnlich war es auch in der Zinkindustrie, wo die vier größten Hütten ebenfalls über 50 v. H. der Produktion auf sich vereinigten ${ }^{117}$. Auch in der Eisenindustrie konzentrierte sich die Produktion nach dem endgültigen Durchbruch der Steinkohlentechnologie; 1875 wurden bereits 96,5 v. H. des Roheisens mittels Steinkohlenkoks erschmolzen ${ }^{118}$, und im gleichen Jahr produzierten die vier größten Hütten 62 v.H. allen Koksroheisens.

Entscheidend für die Behauptung des Einflusses auch unter den Bedingungen industrieller Konzentration war, daß die meisten ihre Industrievermögen in Aktiengesellschaften einbrachten.

1853 machte Guido Henckel von Donnersmarck den Anfang, als er seine Galmeigruben und Zinkhütten in die Schlesische AG für Bergbau und Zinkhüttenbetrieb einbrachte. Die belgische Gesellschaft Vieille Montagne beteiligte sich von

113 Repetzki, Industrie und Wirtschaft, $14 \mathrm{f}$; Knochenhauer, Die oberschlesische Montanindustrie, 110 f.; Popiotek, Górnośląski przemysł, 170-176; Jaros, Historia górnictwa węglowego, 279.

¿14 Kazimierz Popiotek, Koncentracja w przemysłe górniczo-hutniczym Górnego Śląska w drugiej połowie XIX wieku [Die Konzentration in der Bergbau- und Hüttenindustrie Oberschlesiens in der zweiten Hälfte des 19. Jahrhunderts], in: Studia i materiały z dziejów Śląska [Studien und Materialien aus der Geschichte Schlesiens], t. II (1958) 63-181; Fuchs, Vom Dirigismus, 247-258.

115 Popiotek, Górnośląski przemysł, 186-188.

116 Jaros, Historia górnictwa węglowego, 277.

117 Popiotek, Górnośląski przemyst, 70.

118 Ebd. 44. 
Anfang an, und die Gesellschaft entwickelte sich zum größten Zinkproduzenten Europas $^{119}$.

1855 folgte die Minerva aus dem Renardschen Vermögen unter Beteiligung Breslauer und Hamburger Kaufleute und Bankiers.

1871 entstand die Vereinigte Königs- und Laurahütte aus dem Vermögen des Grafen Hugo von Donnersmarck und im gleichen Jahr die Oberschlesische EisenbahnBedarfs AG.

Bis zur Jahrhundertwende folgte noch eine Reihe weiterer Grundherren diesem Beispiel, so daß schließlich mit Ausnahme der profitablen Steinkohlengruben praktisch alle Industriebetriebe aus den adeligen Güterverwaltungen ausgegliedert und in Form von Kapitalgesellschaften betrieben wurden. Damit ging ein weiterer Rückzug der Magnatenfamilien aus der aktiven Geschäftsleitung einher, und die Tätigkeit der Eigentümer beschränkte sich auf die allgemeine Kontrolle, die Bestellung der $\mathrm{Ge}$ schäftsführung und das Einsammeln der Dividenden.

Im frühen 19. Jahrhundert hatte Oberschlesien zweifellos die Führungsrolle bei der Expansion der Montanindustrie übernommen. In den 1830er Jahren wurde dort die Hälfte allen preußischen Roheisens produziert, und auch der Steinkohlenbergbau erlebte einen gewaltigen Aufschwung ${ }^{120}$; die Zinkindustrie war sogar weltweit führend. Gegen Ende des Jahrhunderts hatte Oberschlesien diese Führungsposition im Deutschen Reich längst verloren und gegenüber den anderen deutschen Montanregionen, insbesondere dem Ruhrgebiet, deutlich an Boden verloren. Dies wurde auch von den Zeitgenossen bereits erkannt, die zahlreiche Ursachen für den Verlust der Wettbewerbsfähigkeit anführten, u.a. die Beschränkung des Auslandsabsatzes aufgrund der deutschen Handelspolitik, die günstigere Verkehrslage der anderen Reviere und die dort günstigere Eisenbahntarifpolitik oder die Konkurrenz staatlicher Werke. Moderne Autoren weisen aber auf einen Sachverhalt hin, der eng mit dem eingangs erwähnten Unterschied zwischen kurzfristig-privatem und langfristig-gesamtwirtschaftlichem ökonomischen Erfolg zu tun hat. Jene Logik der Magnatenwirtschaft, die mit billigen Rohstoffen und Arbeitskräften aus den Güterwirtschaften rechnete, minderte über Jahrzehnte den Druck zur Anpassung an effizientere Verfahren und hatte daher eine Verzögerung der technischen Entwicklung zur Folge. Als nun dieser Druck durch Konkurrenz bürgerlicher Gruppen innerhalb Oberschlesiens und nach Aufbrechen der Isolation durch die Eisenbahnen nach außen offenbar wurde, zeigte sich die Rückständigkeit der oberschlesischen Montanindustrie in vollem Umfang. Das immer noch niedrige Lohnniveau ${ }^{121}$ verzögerte eine Mechanisierung und Rationalisierung weiterhin ${ }^{122}$. Hier offenbarte sich das langfristig wirksame Verhängnis der oberschlesischen Magnatenwirtschaft.

\footnotetext{
119 Ebd. $75 \mathrm{f}$.

120 Jaros, Historia górnictwa węglowego, $45 \mathrm{f}$.

121 Stanistaw Kossuth, Górnictwo węglowe na Górnym Śląsku w połowie XIX wieku [Der Kohlenbergbau in Oberschlesien in der Mitte des 19. Jahrhunderts] (Kattowitz 1965) 126.

122 Jaros, Historia górnictwa węglowego, 45 f.; Popiotek, Górnośląski przemysł, 190.
} 


\section{Anhang}

Steinkohlenförderung und Roheisen-, Stabeisen- und Zinkproduktion in Oberschlesien in den Jahren $1800-1850$ (in t)

\begin{tabular}{|c|c|c|c|c|}
\hline Jahr & Steinkohle & Roheisen & Stabeisen & Zink \\
\hline 1800 & 41140 & 17827 & 11207 & - \\
\hline 1801 & 42625 & - & - & - \\
\hline 1802 & 42900 & - & - & - \\
\hline 1803 & 56769 & 19270 & 10838 & - \\
\hline 1804 & 68386 & 22972 & - & - \\
\hline 1805 & 87092 & - & - & - \\
\hline 1806 & 104587 & 16297 & 10626 & - \\
\hline 1807 & 59931 & - & - & - \\
\hline 1808 & 73603 & - & - & - \\
\hline 1809 & 82306 & 17827 & 10918 & 110 \\
\hline 1810 & 93480 & 13516 & 7798 & 140 \\
\hline 1811 & 113760 & 11907 & 8376 & 258 \\
\hline 1812 & 77880 & 14460 & 8506 & 429 \\
\hline 1813 & 75515 & 11122 & 7724 & 216 \\
\hline 1814 & 97574 & - & - & 402 \\
\hline 1815 & 117196 & - & - & 818 \\
\hline 1816 & 147740 & 13560 & 8474 & 1029 \\
\hline 1817 & 147893 & 13310 & 8277 & 1150 \\
\hline 1818 & 175204 & - & - & 1229 \\
\hline 1819 & 153382 & 16880 & 9298 & 1128 \\
\hline 1820 & 146782 & 16482 & 9241 & 1159 \\
\hline 1821 & 165929 & 16299 & 9306 & 1945 \\
\hline 1822 & 204796 & 17913 & 9756 & 3361 \\
\hline 1823 & 34756 & 19017 & 8721 & 7077 \\
\hline 1824 & 344165 & 16424 & 10577 & 10341 \\
\hline 1825 & 360809 & 18423 & 9651 & 12262 \\
\hline 1826 & 304179 & 19630 & 11433 & 9777 \\
\hline 1827 & 327894 & 22379 & 11473 & 11240 \\
\hline 1828 & 281047 & 20812 & 13838 & 9290 \\
\hline 1829 & 250318 & 22094 & 14870 & 6746 \\
\hline 1830 & 217435 & 22660 & 13603 & 4681 \\
\hline 1831 & 186678 & 22723 & 15410 & 5226 \\
\hline 1832 & 250480 & 25348 & 20074 & 5289 \\
\hline 1833 & 267464 & 26324 & 15522 & 5433 \\
\hline 1834 & 268938 & 24919 & 15408 & 6491 \\
\hline 1835 & 306832 & - & - & 8755 \\
\hline 1836 & 338473 & 26962 & 17132 & 11576 \\
\hline 1837 & 385065 & 30647 & 18402 & 10686 \\
\hline 1838 & 423367 & 32005 & 18091 & 10496 \\
\hline 1839 & 440864 & 33321 & 23184 & 10712 \\
\hline 1840 & 538556 & 37602 & 24190 & 10232 \\
\hline 1841 & 568713 & 40249 & 24829 & 10004 \\
\hline 1842 & 612974 & 36333 & 24834 & 12987 \\
\hline 1843 & 619068 & 36395 & 26418 & 16650 \\
\hline 1846 & 674618 & 35934 & 28807 & 18922 \\
\hline 1845 & 818992 & 36649 & 38521 & 19650 \\
\hline 1846 & 867190 & 50028 & 38112 & 19827 \\
\hline 1847 & 901408 & 45605 & 36260 & 20503 \\
\hline 1848 & 873707 & 44289 & 29414 & 18620 \\
\hline 1849 & 915936 & 42660 & 30996 & 23282 \\
\hline 1850 & 975401 & 52200 & 39176 & 25518 \\
\hline
\end{tabular}

Quelle: Zbigniew Kwaśny, Rozwój przemysłu 
